\title{
A Note on Sign-Changing Solutions to the NLS on the Double-Bridge Graph
}

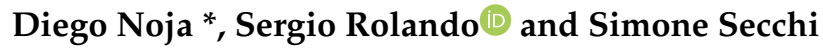 \\ Dipartimento di Matematica e Applicazioni, Università di Milano Bicocca, via R. Cozzi 55, 20126 Milano, Italy; \\ sergio.rolando@unimib.it (S.R.); simone.secchi@unimib.it (S.S.) \\ * Correspondence: diego.noja@unimib.it
}

Received: 15 January 2019; Accepted: 28 January 2019; Published: 1 February 2019

check for updates

\begin{abstract}
We study standing waves of the NLS equation posed on the double-bridge graph: two semi-infinite half-lines attached at a circle. At the two vertices, Kirchhoff boundary conditions are imposed. We pursue a recent study concerning solutions nonzero on the half-lines and periodic on the circle, by proving some existing results of sign-changing solutions non-periodic on the circle.
\end{abstract}

Keywords: quantum graphs; non-linear Schrödinger equation; standing waves

MSC: 35Q55; 81Q35; 35R02

\section{Introduction and Main Results}

The study of nonlinear equations on graphs, especially the nonlinear Schrödinger equation (NLS), is a quite recent research subject, which already produced a plenty of interesting results (see [1-3]). The attractive feature of these mathematical models is the complexity allowed by the graph structure, joined with the one dimensional character of the equations. While they are an oversimplification in many real problems, they appear indicative of several dynamically interesting phenomena that are atypical or unexpected in more standard frameworks. The most studied issue concerning NLS is certainly the existence and characterization of standing waves (see, e.g., [4-9]). More particularly, several results are known about ground states (standing waves of minimal energy at fixed mass, i.e., $L^{2}$ norm) as regard existence, non-existence and stability properties, depending on various characteristics of the graph [2,10-13].

In this paper, we are interested in a special example, which reveals an unsuspectedly complex structure of the set of standing waves. More precisely, we consider a metric graph $\mathcal{G}$ made up of two half lines joined by two bounded edges, i.e., a so-called double-bridge graph (see Figure 1). $\mathcal{G}$ can also be thought of as a ring with two half lines attached in two distinct vertices. The half lines are both identified with the interval $[0,+\infty)$, while the bounded edges are represented by two bounded intervals of lengths $L_{1}>0$ and $L_{2} \geq L_{1}$, precisely $\left[0, L_{1}\right]$ and $\left[L_{1}, L\right]$ with $L=L_{1}+L_{2}$.

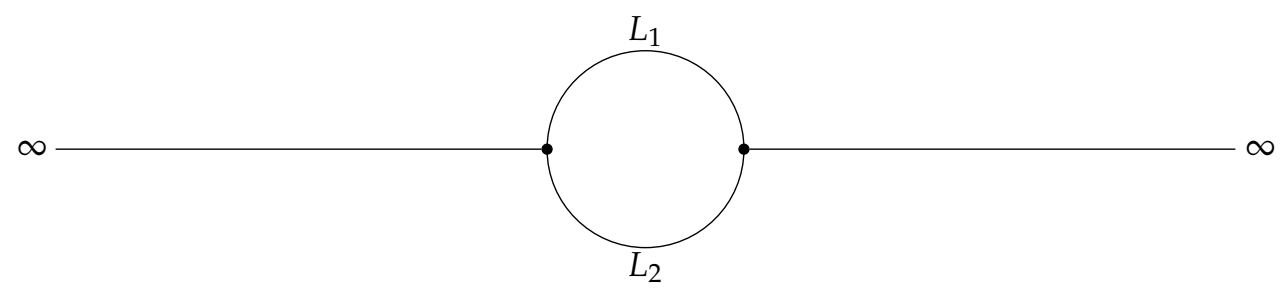

Figure 1. The double-bridge graph. 
A function $\psi$ on $\mathcal{G}$ is a Cartesian product $\psi\left(x_{1}, \ldots, x_{4}\right)=\left(\psi_{1}\left(x_{1}\right), \ldots, \psi_{4}\left(x_{4}\right)\right)$ with $x_{j} \in I_{j}$ for $j=1, \ldots, 4$, where $I_{1}=\left[0, L_{1}\right], I_{2}=\left[L_{1}, L\right]$ and $I_{3}=I_{4}=[0,+\infty)$. Then, a Schrödinger operator $H_{\mathcal{G}}$ on $\mathcal{G}$ is defined as

$$
H_{\mathcal{G}} \psi\left(x_{1}, \ldots, x_{4}\right)=\left(-\psi_{1}^{\prime \prime}\left(x_{1}\right), \ldots,-\psi_{4}^{\prime \prime}\left(x_{4}\right)\right), \quad x_{j} \in I_{j},
$$

with domain $D\left(H_{\mathcal{G}}\right)$ given by the functions $\psi$ on $\mathcal{G}$ whose components satisfy $\psi_{j} \in H^{2}\left(I_{j}\right)$ together with the so-called Kirchhoff boundary conditions, i.e.,

$$
\begin{gathered}
\psi_{1}(0)=\psi_{2}(L)=\psi_{3}(0), \quad \psi_{1}\left(L_{1}\right)=\psi_{2}\left(L_{1}\right)=\psi_{4}(0), \\
\psi_{1}^{\prime}(0)-\psi_{2}^{\prime}(L)+\psi_{3}^{\prime}(0)=\psi_{1}^{\prime}\left(L_{1}\right)-\psi_{2}^{\prime}\left(L_{1}\right)-\psi_{4}^{\prime}(0)=0 .
\end{gathered}
$$

As is well known (see [14] for general information on quantum graphs), the operator $H_{\mathcal{G}}$ is self-adjoint on the domain $D\left(H_{\mathcal{G}}\right)$, and it generates a unitary Schrödinger dynamics. Essential information about its spectrum is given in ([15], Appendix A). We perturb this linear dynamics with a focusing cubic term, namely we consider the following NLS on $\mathcal{G}$

$$
i \frac{d \psi_{t}}{d t}=H_{\mathcal{G}} \psi_{t}-\left|\psi_{t}\right|^{2} \psi_{t}
$$

where the nonlinear term $\left|\psi_{t}\right|^{2} \psi_{t}$ is a shortened notation for $\left(\left|\psi_{1, t}\right|^{2} \psi_{1, t}, \ldots,\left|\psi_{4, t}\right|^{2} \psi_{4, t}\right)$. Hence, Equation (4) is a system of scalar NLS equations on the intervals $I_{j}$ coupled through the Kirchhoff boundary conditions in Equations (2)-(3) included in the domain of $H_{\mathcal{G}}$. On rather general grounds, it can be shown that this problem enjoys well-posedness both in strong sense and in the energy space (see in particular ([2], Section 2.6)).

We are interested in standing waves of Equation (4), i.e., its solutions of the form $\psi_{t}=e^{-i \omega t} U(x)$ where $\omega \in \mathbb{R}$ and $U\left(x_{1}, \ldots, x_{4}\right)=\left(u_{1}\left(x_{1}\right), \ldots, u_{4}\left(x_{4}\right)\right)$ is a purely spatial function on $\mathcal{G}$, which may also depend on $\omega$. Such a problem has already been considered in $[11,12,15,16]$. In particular, in [11,12], variational methods are used to show, among many other things, that Equation (4) has no ground state, i.e., no standing wave exists that minimizes the energy at fixed $L^{2}$-norm. In a recent paper [16], information on positive bound states that are not ground states is given. The special example of tadpole graph (a ring with a single half-line) is treated in detail in $[17,18]$.

As for the results in [15], they can be summarized as follows. Writing the problem of standing waves of Equation (4) component-wise, we get the following scalar problem:

$$
\begin{cases}-u_{j}^{\prime \prime}-u_{j}^{3}=\omega u_{j}, & u_{j} \in H^{2}\left(I_{j}\right) \\ u_{1}(0)=u_{2}(L)=u_{3}(0), & u_{1}\left(L_{1}\right)=u_{2}\left(L_{1}\right)=u_{4}(0) \\ u_{1}^{\prime}(0)-u_{2}^{\prime}(L)+u_{3}^{\prime}(0)=0, & u_{1}^{\prime}\left(L_{1}\right)-u_{2}^{\prime}\left(L_{1}\right)-u_{4}^{\prime}(0)=0 .\end{cases}
$$

Such a system has solutions with $u_{3}=u_{4}=0$ if and only if the ratio $L_{1} / L_{2}$ is rational. In this case, they form a sequence of continuous branches in the $\left(\omega,\|U\|_{L^{2}}\right)$ plane, bifurcating from the linear eigenvectors of the Schrödinger operator $H_{\mathcal{G}}$ (see Figure 2), and they are periodic on the ring of $\mathcal{G}$, that is, $u_{1}$ and $u_{2}$ are restrictions to $I_{1}$ and $I_{2}$ of a function $u$ belonging to the second Sobolev space of periodic functions $H_{\text {per }}^{2}([0, L])=\left\{u \in H^{2}([0, L]): u(0)=u(L), u^{\prime}(0)=u^{\prime}(L)\right\}$. In particular, such function $u$ is a rescaled Jacobi cnoidal function (see, e.g., $[19,20]$ for a treatise on the Jacobian elliptic functions). If $\omega \geq 0$, no other nonzero standing waves exist, since the NLS on the unbounded edges has no nontrivial solution. If $\omega<0$, instead, the NLS on the half lines has soliton solutions, so that standing waves with nonzero $u_{3}$ and $u_{4}$ are admissible. The general study of this kind of solutions leads to a rather complicated system of equations, since, while $u_{3}$ and $u_{4}$ must be shifted solitons, each of $u_{1}$ and $u_{2}$ can be (at least in principle) a cnoidal function, a dnoidal function or a shifted soliton. To limit this complexity, the analysis in [15] is focused on the special case of standing 
waves that are non-vanishing on the half lines but share the above-mentioned periodicity feature with the bifurcation solutions. This amounts to study the following system:

$$
\left\{\begin{array}{l}
-u^{\prime \prime}-u^{3}=\omega u, \quad u \in H_{\text {per }}^{2}([0, L]), \omega<0 \\
u(0)= \pm u\left(L_{1}\right)=\sqrt{2|\omega|}
\end{array}\right.
$$

where the sign \pm distinguishes the cases of $u_{3}$ and $u_{4}$ with the same sign (which we may assume positive, thanks to the odd parity of the equation) or with different signs. In [15], it is shown that:

(i) If $L_{1} / L_{2} \in \mathbb{Q}$, then the set of solutions to (6) is made up of a sequence of secondary bifurcation branches $\left\{\left(\omega, \widetilde{u}_{n, \omega}\right): \omega<0\right\}_{n>1}$, originating at $\omega=0$ from each of the previous ones, together with a sequence $\left\{\left(\omega_{n}, u_{n}\right)\right\}_{n \geq 1}$ not lying on any branch (see Figure 2).

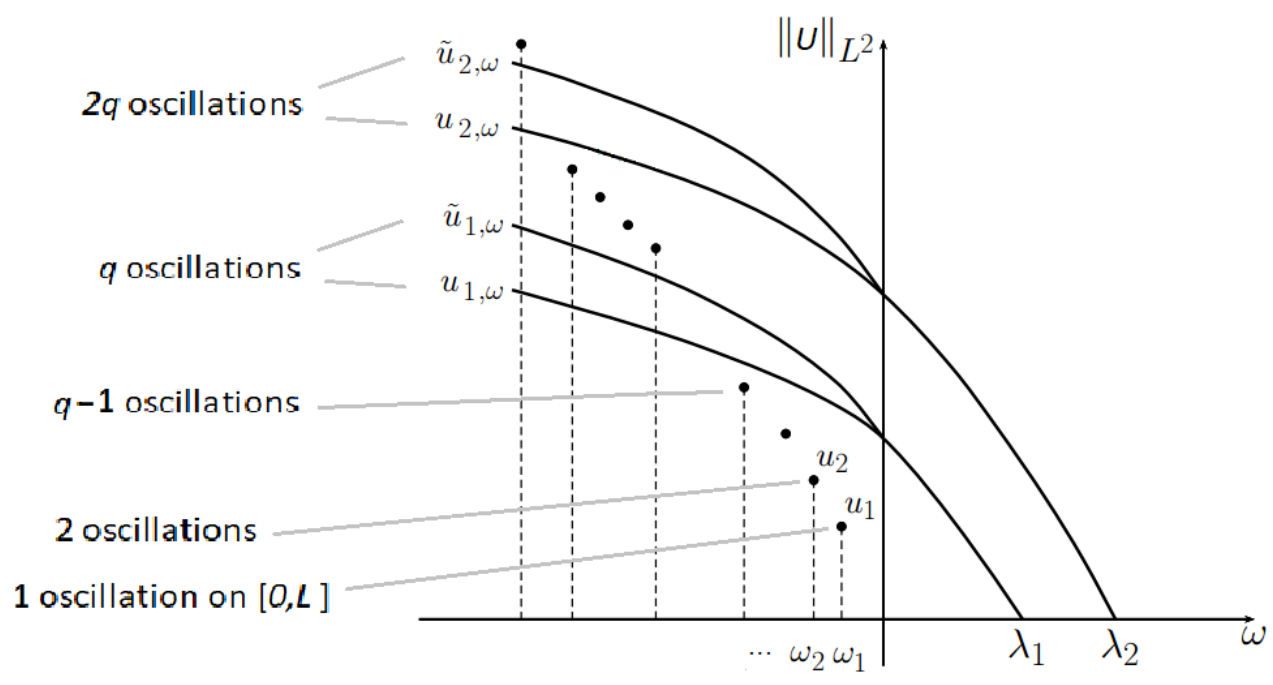

Figure 2. Bifurcation diagram for $L_{1} / L=p / q$ with $p, q \in \mathbb{N}$ coprime.

(ii) If $L_{1} / L_{2} \notin \mathbb{Q}$, then the set of solutions to (6) reduces to two sequences $\left\{\left(\omega_{n}^{+}, u_{n}^{+}\right)\right\}_{n \geq 1}$ and $\left\{\left(\omega_{n}^{-}, u_{n}^{-}\right)\right\}_{n \geq 1}$ alone, solving the problem in Equation (6) with sign \pm , respectively, where the frequency sequences $\left\{\omega_{n}^{ \pm}\right\}_{n \geq 1}$ are unbounded below and have at least a finite nonzero cluster point (see Figure 3). The functions $u_{n}^{ \pm}$oscillate $n$ times on the ring of the graph.

These results come rather unexpectedly, so the aim of this paper is to pursue the study begun in [15] by deepening the understanding of such results in relation to the underlying physical model. In particular, we ask the following questions: Does Equation (4) admit standing waves that are non-periodic on ring of $\mathcal{G}$ ? If so, do they form continuous branches to which the isolated periodic solutions belong? 


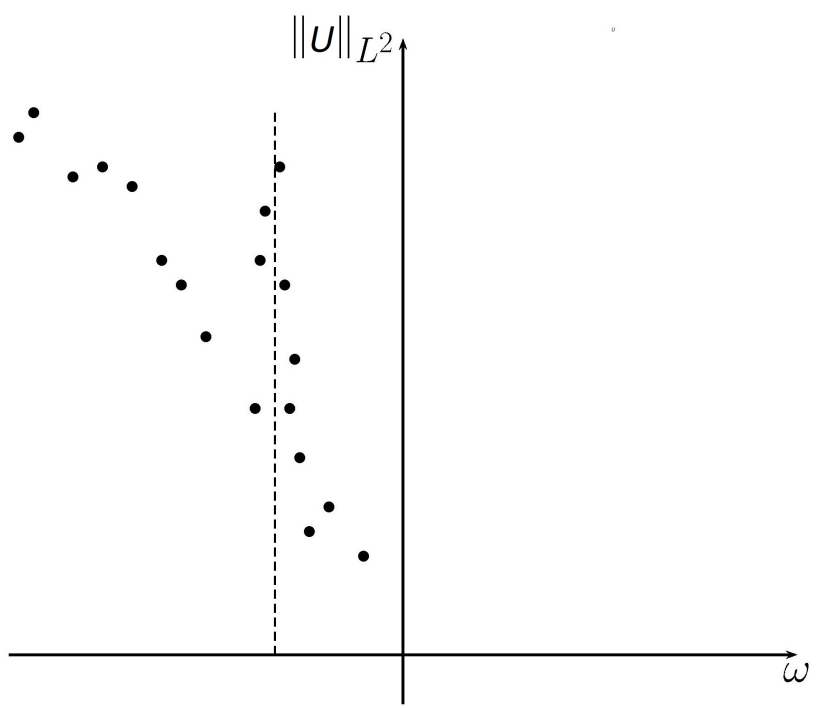

Figure 3. The appearance of each of the sequences $\left\{\left(\omega_{n}^{+}, u_{n}^{+}\right)\right\}_{n \in \mathbb{N}}$ and $\left\{\left(\omega_{n}^{-}, u_{n}^{-}\right)\right\}_{n \in \mathbb{N}}$ for $L_{1} / L \in \mathbb{R} \backslash \mathbb{Q}$.

With a view to especially answer the second question, we look for standing waves which include the ones given by Equation (6) but still change sign on the bounded edges. More precisely, we look for solutions to Equation (5) exhibiting the following features:

- $u_{1}, u_{2}$ are sign-changing.

- $u_{3}, u_{4}$ are nonzero.

The second feature implies $\omega<0$ and

$$
u_{j}(x)= \pm \sqrt{2} \eta \operatorname{sech}\left(\eta\left(x+a_{j}\right)\right), \quad a_{j} \in \mathbb{R}, j=3,4
$$

where we set $\eta:=\sqrt{|\omega|}$ for brevity. Then, the first feature implies

$$
u_{j}(x)=\eta \sqrt{\frac{2 k_{j}^{2}}{2 k_{j}^{2}-1}} \text { cn }\left(\frac{\eta}{\sqrt{2 k_{j}^{2}-1}}\left(x+a_{j}\right) ; k_{j}\right), \quad k_{j} \in\left(\frac{1}{\sqrt{2}}, 1\right), a_{j} \in\left[0, T_{j}\right), j=1,2
$$

where $\mathrm{cn}(\cdot ; k)$ is the cnoidal function of parameter $k$ and $T_{j}=T_{j}\left(k_{j}, \eta\right):=S\left(k_{j}\right) / \eta$ is the period of the function $\mathrm{cn}\left(\eta(\cdot) / \sqrt{2 k_{j}^{2}-1} ; k_{j}\right)$. Here and in the rest of the paper, $S$ denotes the function

$$
S(k):=4 \sqrt{2 k^{2}-1} K(k)=4 \sqrt{2 k^{2}-1} \int_{0}^{1} \frac{d t}{\sqrt{\left(1-t^{2}\right)\left(1-k^{2} t^{2}\right)}},
$$

where $K(k)$ is the so called complete elliptic integral of first kind. Notice that $S:(1 / \sqrt{2}, 1) \rightarrow \mathbb{R}$ is strictly increasing, continuous and such that $S((1 / \sqrt{2}, 1))=(0,+\infty)$.

Therefore, restricting ourselves for simplicity to the case with $u_{3}$ and $u_{4}$ of the same sign, which we may assume positive thanks to the odd parity of the system in Equation (5), we are led to study the existence of solutions $\eta>0, k_{1}, k_{2} \in\left(\frac{1}{\sqrt{2}}, 1\right), a_{1} \in\left[0, T_{1}\right), a_{2} \in\left[0, T_{2}\right), a_{3}, a_{4} \in \mathbb{R}$ to the following system: 


$$
\left\{\begin{array}{l}
\frac{k_{1}}{\sqrt{2 k_{1}^{2}-1}} \mathrm{cn}\left(\frac{\eta a_{1}}{\sqrt{2 k_{1}^{2}-1}} ; k_{1}\right)=\frac{k_{2}}{\sqrt{2 k_{2}^{2}-1}} \mathrm{cn}\left(\frac{\eta\left(L+a_{2}\right)}{\sqrt{2 k_{2}^{2}-1}} ; k_{2}\right)=\operatorname{sech}\left(\eta a_{3}\right) \\
\frac{k_{1}}{\sqrt{2 k_{1}^{2}-1}} \mathrm{cn}\left(\frac{\eta\left(L_{1}+a_{1}\right)}{\sqrt{2 k_{1}^{2}-1}} ; k_{1}\right)=\frac{k_{2}}{\sqrt{2 k_{2}^{2}-1}} \mathrm{cn}\left(\frac{\eta\left(L_{1}+a_{2}\right)}{\sqrt{2 k_{2}^{2}-1}} ; k_{2}\right)=\operatorname{sech}\left(\eta a_{4}\right) \\
\tanh \left(\eta a_{3}\right) \operatorname{sech}\left(\eta a_{3}\right)= \\
=-\frac{k_{1}}{2 k_{1}^{2}-1} \operatorname{sn}\left(\frac{\eta a_{1}}{\sqrt{2 k_{1}^{2}-1}} ; k_{1}\right) \mathrm{dn}\left(\frac{\eta a_{1}}{\sqrt{2 k_{1}^{2}-1}} ; k_{1}\right)+\frac{k_{2}}{2 k_{2}^{2}-1} \operatorname{sn}\left(\frac{\eta\left(L+a_{2}\right)}{\sqrt{2 k_{2}^{2}-1}} ; k_{2}\right) \mathrm{dn}\left(\frac{\eta\left(L+a_{2}\right)}{\sqrt{2 k_{2}^{2}-1}} ; k_{2}\right) \\
\tanh \left(\eta a_{4}\right) \operatorname{sech}\left(\eta a_{4}\right)= \\
=\frac{k_{1}}{2 k_{1}^{2}-1} \operatorname{sn}\left(\frac{\eta\left(L_{1}+a_{1}\right)}{\sqrt{2 k_{1}^{2}-1}} ; k_{1}\right) \mathrm{dn}\left(\frac{\eta\left(L_{1}+a_{1}\right)}{\sqrt{2 k_{1}^{2}-1}} ; k_{1}\right)-\frac{k_{2}}{2 k_{2}^{2}-1} \operatorname{sn}\left(\frac{\eta\left(L_{1}+a_{2}\right)}{\sqrt{2 k_{2}^{2}-1}} ; k_{2}\right) \mathrm{dn}\left(\frac{\eta\left(L_{1}+a_{2}\right)}{\sqrt{2 k_{2}^{2}-1}} ; k_{2}\right) .
\end{array}\right.
$$

This set of equations turns out to be still rather difficult to study in his full generality, and indeed we have results only in the subcase where the two solitons in Equation (7) have the same height at the vertices, i.e., sech $\left(\eta a_{3}\right)=\operatorname{sech}\left(\eta a_{4}\right)$ (which corresponds to $\theta_{1}=\theta_{2}$ in Section 2 ). More precisely, in Section 2 we reduce the system in Equation (10) to an equivalent one, which naturally splits into different cases. Then, we study three of such cases, all with sech $\left(\eta a_{3}\right)=\operatorname{sech}\left(\eta a_{4}\right)$, leading to our existence results, which are the following three theorems.

The first two results only concern the case of irrational ratios $L_{1} / L_{2}$ and give solutions with $k_{1} \neq k_{2}$, i.e., non-periodic on the ring of the graph.

Theorem 1. Assume that $L_{1} / L_{2} \in \mathbb{R} \backslash \mathbb{Q}$. Then, there exists a sequence of positive integers $\left(n_{h}\right)_{h \in \mathbb{N}}$ such that for every $\omega<-32 K(1 / \sqrt{2})^{2} /\left(L_{1} L_{2}\right)$ there exists $h_{\omega} \in \mathbb{N}$ (also depending on $L_{1}$ and $\left.L_{2}\right)$ such that for all $h>h_{\omega}$ the problem in Equation (5) has two solutions $\left(u_{1, h}^{+}, u_{2, h}^{+}, u_{3, h}^{+}, u_{4, h}^{+}\right)$and $\left(u_{1, h}^{-}, u_{2, h}^{-}, u_{3, h}^{-}, u_{4, h}^{-}\right)$of the form:

$$
\begin{gathered}
u_{j, h}^{ \pm}(x)=\sqrt{\frac{2|\omega| k_{j, h}^{2}}{2 k_{j, h}^{2}-1}} \text { cn }\left(\sqrt{\frac{|\omega|}{2 k_{j, h}^{2}-1}}\left(x+a_{j, h}^{ \pm}\right) ; k_{j, h}\right), \quad j=1,2 \\
u_{j, h}^{ \pm}(x)=\sqrt{2|\omega|} \operatorname{sech}\left(\sqrt{|\omega|}\left(x+a_{j, h}^{ \pm}\right)\right), \quad j=3,4
\end{gathered}
$$

where $u_{1, h}^{ \pm}(x)$ and $u_{2, h}^{ \pm}(x)$ have periods $T_{1, h}=L_{1} /\left[n_{h} L_{1} / L_{2}+1\right]$ and $T_{2, h}=L_{2} / n_{h}$, and for all $h$ one has

$$
\frac{1}{\sqrt{2}}<k_{1, h}<k_{2, h}<1, \quad a_{1, h}^{ \pm} \in\left(0, \frac{T_{1, h}}{4}\right), \quad a_{2, h}^{ \pm} \in\left[0, T_{2, h}\right), \quad a_{3, h}^{ \pm}<0, \quad a_{4, h}^{ \pm}>0, \quad a_{j, h}^{+} \neq a_{j, h}^{-} .
$$

Remark 1. More precisely, according to the proof, in Theorem 1, we have that

$$
\begin{aligned}
& k_{1, h}=S^{-1}\left(\frac{L_{1}}{\left[n_{h} L_{1} / L_{2}+1\right]} \sqrt{|\omega|}\right), \quad a_{1, h}^{ \pm}=\gamma_{1}\left(k_{1, h}, \omega, \theta_{h}^{ \pm}\right), \\
& k_{2, h}=S^{-1}\left(\frac{L_{2}}{n_{h}} \sqrt{|\omega|}\right), \quad a_{2, h}^{ \pm}=\gamma_{2}\left(k_{2, h}, \omega, \theta_{h}^{ \pm}\right)-L+p T_{2, h}, \quad-a_{3, h}^{ \pm}=a_{4, h}^{ \pm}=\operatorname{sech}_{\mid[0,+\infty)}^{-1}\left(\theta_{h}^{ \pm}\right),
\end{aligned}
$$

where $p$ is the unique positive integer such that $a_{2, h}^{ \pm} \in\left[0, T_{2, h}\right), \theta_{h}^{ \pm}$are the two distinct solutions in $(0,1]$ of the equation $\theta^{2}\left(1-\theta^{2}\right)=t_{k_{1, h}, k_{2, h}}$ with $t_{k_{1, h}, k_{2, h}}$ given by Equation (17), and $\gamma_{j}\left(k_{j, h}, \omega, \theta_{h}^{ \pm}\right)$is the unique preimage in $\left(0, T_{j, h} / 4\right)$ of $\theta_{h}^{ \pm} \sqrt{2 k_{j, h}^{2}-1} / k_{j, h}$ by the function cn $\left((\cdot) \sqrt{|\omega|} / \sqrt{2 k_{j, h}^{2}-1} ; k_{j, h}\right)$.

Theorem 2. Assume that $L_{1} / L_{2} \in \mathbb{R} \backslash \mathbb{Q}$. Then, there exists a sequence of positive integers $\left(n_{h}\right)_{h \in \mathbb{N}}$ such that for every $\omega<-32 K(1 / \sqrt{2})^{2} /\left(L_{1} L_{2}\right)$ there exists $h_{\omega} \in \mathbb{N}$ (also depending on $L_{1}$ and $L_{2}$ ) such that for all $h>h_{\omega}$ the problem in Equation (5) has two solutions $\left(u_{1, h}^{ \pm}, u_{2, h}^{ \pm}, u_{3, h}^{ \pm}, u_{4, h}^{ \pm}\right)$of the form of Equations (11)-(12), where $u_{1, h}^{ \pm}(x)$ and $u_{2, h}^{ \pm}(x)$ have periods $T_{1, h}=L_{1} /\left[n_{h} L_{1} / L_{2}\right]$ and $T_{2, h}=L_{2} / n_{h}$, the parameters $a_{1, h}^{ \pm}, a_{2, h}^{ \pm}, a_{3, h}^{ \pm}, a_{4, h}^{ \pm}$are as in Equation (13) and for all $h$ one has 


$$
\frac{1}{\sqrt{2}}<k_{2, h}<k_{1, h}<1
$$

Remark 2. More precisely, in Theorem 2 we have that

$$
k_{1, h}=S^{-1}\left(\frac{L_{1}}{\left[n_{h} L_{1} / L_{2}\right]} \sqrt{|\omega|}\right) \text { and } k_{2, h}=S^{-1}\left(\frac{L_{2}}{n_{h}} \sqrt{|\omega|}\right),
$$

whereas $a_{j, h}^{ \pm}$are exactly as in Remark 1 .

The third result does not need $L_{1} / L_{2}$ irrational and concerns the subcase of the system in Equation (5) which, if $L_{1} / L_{2} \in \mathbb{R} \backslash \mathbb{Q}$ and $k_{1}=k_{2}$, is exactly the system in Equation (6) with plus sign (see Remark 5).

Theorem 3. Let $m, n \in \mathbb{N}$ be such that $n>m \geq 1$. Then, there exists $\omega_{m, n}<0$ (also depending on $L_{1}$ ) such that for all $\omega<\omega_{m, n}$ the problem in Equation (5) has a solution $\left(u_{1}, u_{2}, u_{3}, u_{4}\right)$ of the form of Equations (7)-(8), with $k_{1}, k_{2} \in(\sqrt{3} / 2,1), a_{1} \in\left(0, T_{1} / 4\right), a_{2} \in\left[0, T_{2}\right)$.

Remark 3. According to the proof, in Theorem $3, a_{1}, a_{2}, a_{3}, a_{4}$ can be described in a similar way of Theorems 1 and 2. On the contrary, the parameters $k_{1}, k_{2}$ do exist, but are not explicit as in the previous theorems.

As already mentioned, Theorems 1-3 do not exhaust the study of solutions to the problem in Equation (5), and thus of standing waves of (NLS), as they only concern the case of solitons having the same height at the vertices. In addition, they do not describe the whole family of this kind of solutions, but only give existence results. However, they still provide some answer to the questions raised above. Indeed, Theorems 1 and 2 answer in the affirmative to the first question, as they prove existence of standing waves which are non-periodic on the ring of $\mathcal{G}$. As to Theorem 3 , for any $m$ and $n$, it provides a family of solutions which depend on the continuous parameter $\omega \in\left(-\infty, \omega_{m, n}\right)$ and, roughly speaking, make $m$ oscillations on the edge of length $L_{1}$ and $n-m$ oscillations on the one of length $L_{2}$ (cf. the second and third equations of the system in Equation (33)). If $L_{1} / L_{2}$ is irrational and one of these families contain a solution with $k_{1}=k_{2}$, then such a solution is one of the isolated solutions found in [15] in the irrational case and we can answer affirmatively also to the second question. Unfortunately, the argument we used in proving Theorem 3 does not allow us to say wether we find solutions with $k_{1}=k_{2}$ or not, and therefore we do not have a final answer to the second question.

\section{Preliminaries}

In this section, we reduce the system in Equation (10) to a simpler equivalent one, which is the system in Equation (14) with the last two equations replaced by the system in Equation (19).

For brevity, we set

$$
X_{1}=\frac{\eta a_{1}}{\sqrt{2 k_{1}^{2}-1}}, \quad X_{2}=\frac{\eta\left(L+a_{2}\right)}{\sqrt{2 k_{2}^{2}-1}}, \quad X_{3}=\frac{\eta\left(L_{1}+a_{1}\right)}{\sqrt{2 k_{1}^{2}-1}}, \quad X_{4}=\frac{\eta\left(L_{1}+a_{2}\right)}{\sqrt{2 k_{2}^{2}-1}},
$$

and

$$
\sigma_{1}=\operatorname{sgn}\left[\operatorname{sn}\left(X_{1} ; k_{1}\right)\right], \quad \sigma_{2}=\operatorname{sgn}\left[\operatorname{sn}\left(X_{2} ; k_{2}\right)\right], \quad \sigma_{3}=\operatorname{sgn}\left[\operatorname{sn}\left(X_{3} ; k_{1}\right)\right], \quad \sigma_{4}=\operatorname{sgn}\left[\operatorname{sn}\left(X_{4} ; k_{2}\right)\right] .
$$

Then, using well known identities (see [20]) and the first equation of the system in Equation (10), we get 


$$
\begin{aligned}
& \operatorname{sn}\left(X_{1} ; k_{1}\right)=\sigma_{1} \sqrt{1-\mathrm{cn}^{2}\left(X_{1} ; k_{1}\right)}=\sigma_{1} \sqrt{1-\frac{2 k_{1}^{2}-1}{k_{1}^{2}} \operatorname{sech}^{2}\left(\eta a_{3}\right)}, \\
& \operatorname{dn}\left(X_{1} ; k_{1}\right)=\sqrt{1-k_{1}^{2}+k_{1}^{2} \mathrm{cn}^{2}\left(X_{1} ; k_{1}\right)}=\sqrt{1-k_{1}^{2}+\left(2 k_{1}^{2}-1\right) \operatorname{sech}^{2}\left(\eta a_{3}\right)}
\end{aligned}
$$

and hence

$$
\begin{aligned}
\frac{k_{1}}{2 k_{1}^{2}-1} \operatorname{sn}\left(X_{1} ; k_{1}\right) \operatorname{dn}\left(X_{1} ; k_{1}\right) & =\sigma_{1} \sqrt{\frac{k_{1}^{2}}{2 k_{1}^{2}-1}-\operatorname{sech}^{2}\left(\eta a_{3}\right)} \sqrt{\frac{\left(1-k_{1}^{2}\right)}{2 k_{1}^{2}-1}+\operatorname{sech}^{2}\left(\eta a_{3}\right)} \\
& =\sigma_{1} \sqrt{\frac{k_{1}^{2}\left(1-k_{1}^{2}\right)}{\left(2 k_{1}^{2}-1\right)^{2}}+\operatorname{sech}^{2}\left(\eta a_{3}\right)-\operatorname{sech}^{4}\left(\eta a_{3}\right) .}
\end{aligned}
$$

Arguing similarly for the products $\operatorname{sn}\left(X_{2} ; k_{2}\right) \operatorname{dn}\left(X_{2} ; k_{2}\right), \operatorname{sn}\left(X_{3} ; k_{1}\right) \operatorname{dn}\left(X_{3} ; k_{1}\right)$ and $\operatorname{sn}\left(X_{4} ; k_{2}\right) \mathrm{dn}\left(X_{4} ; k_{2}\right)$, and defining

$$
c(k):=\frac{k^{2}\left(1-k^{2}\right)}{\left(2 k^{2}-1\right)^{2}},
$$

we thus obtain that the system in Equation (10) is equivalent to

$$
\left\{\begin{array}{l}
\frac{k_{1}}{\sqrt{2 k_{1}^{2}-1}} \operatorname{cn}\left(\frac{\eta a_{1}}{\sqrt{2 k_{1}^{2}-1}} ; k_{1}\right)=\frac{k_{2}}{\sqrt{2 k_{2}^{2}-1}} \operatorname{cn}\left(\frac{\eta\left(L+a_{2}\right)}{\sqrt{2 k_{2}^{2}-1}} ; k_{2}\right)=\operatorname{sech}\left(\eta a_{3}\right) \\
\frac{k_{1}}{\sqrt{2 k_{1}^{2}-1}} \operatorname{cn}\left(\frac{\eta\left(L_{1}+a_{1}\right)}{\sqrt{2 k_{1}^{2}-1}} ; k_{1}\right)=\frac{k_{2}}{\sqrt{2 k_{2}^{2}-1}} \operatorname{cn}\left(\frac{\eta\left(L_{1}+a_{2}\right)}{\sqrt{2 k_{2}^{2}-1}} ; k_{2}\right)=\operatorname{sech}\left(\eta a_{4}\right) \\
\tanh \left(\eta a_{3}\right) \operatorname{sech}\left(\eta a_{3}\right)=-\sigma_{1} \sqrt{c\left(k_{1}\right)+\operatorname{sech}^{2}\left(\eta a_{3}\right)-\operatorname{sech}^{4}\left(\eta a_{3}\right)}+\sigma_{2} \sqrt{c\left(k_{2}\right)+\operatorname{sech}^{2}\left(\eta a_{3}\right)-\operatorname{sech}^{4}\left(\eta a_{3}\right)} \\
\tanh \left(\eta a_{4}\right) \operatorname{sech}\left(\eta a_{4}\right)=\sigma_{3} \sqrt{c\left(k_{1}\right)+\operatorname{sech}^{2}\left(\eta a_{4}\right)-\operatorname{sech}^{4}\left(\eta a_{4}\right)}-\sigma_{4} \sqrt{c\left(k_{2}\right)+\operatorname{sech}^{2}\left(\eta a_{4}\right)-\operatorname{sech}^{4}\left(\eta a_{4}\right) .}
\end{array}\right.
$$

Let us now focus on the last two equations. Setting

$$
\theta_{1}=\operatorname{sech}\left(\eta a_{3}\right), \theta_{2}=\operatorname{sech}\left(\eta a_{4}\right), \sigma_{5}=\operatorname{sgn}\left(a_{3}\right)=\operatorname{sgn}\left(\tanh \left(\eta a_{3}\right)\right), \sigma_{6}=\operatorname{sgn}\left(a_{4}\right)=\operatorname{sgn}\left(\tanh \left(\eta a_{4}\right)\right)
$$

the couple of such equations is equivalent to

$$
\left\{\begin{array}{l}
\sigma_{5} \sqrt{1-\theta_{1}^{2}} \theta_{1}=-\sigma_{1} \sqrt{c\left(k_{1}\right)+\theta_{1}^{2}\left(1-\theta_{1}^{2}\right)}+\sigma_{2} \sqrt{c\left(k_{2}\right)+\theta_{1}^{2}\left(1-\theta_{1}^{2}\right)} \\
\sigma_{6} \sqrt{1-\theta_{2}^{2}} \theta_{2}=\sigma_{3} \sqrt{c\left(k_{1}\right)+\theta_{2}^{2}\left(1-\theta_{2}^{2}\right)}-\sigma_{4} \sqrt{c\left(k_{2}\right)+\theta_{2}^{2}\left(1-\theta_{2}^{2}\right)} .
\end{array}\right.
$$

Squaring the equations, we get

$$
\begin{aligned}
& c\left(k_{1}\right)+\theta_{1}^{2}\left(1-\theta_{1}^{2}\right)+c\left(k_{2}\right)-2 \sigma_{1} \sigma_{2} \sqrt{c\left(k_{1}\right)+\theta_{1}^{2}\left(1-\theta_{1}^{2}\right)} \sqrt{c\left(k_{2}\right)+\theta_{1}^{2}\left(1-\theta_{1}^{2}\right)}=0, \\
& c\left(k_{1}\right)+\theta_{2}^{2}\left(1-\theta_{2}^{2}\right)+c\left(k_{2}\right)-2 \sigma_{3} \sigma_{4} \sqrt{c\left(k_{1}\right)+\theta_{2}^{2}\left(1-\theta_{2}^{2}\right)} \sqrt{c\left(k_{2}\right)+\theta_{2}^{2}\left(1-\theta_{2}^{2}\right)}=0,
\end{aligned}
$$

which are impossible if $\sigma_{1} \sigma_{2}=-1$ or $\sigma_{3} \sigma_{4}=-1$. Hence, we can add the conditions $\sigma_{1}=\sigma_{2}$ and $\sigma_{3}=\sigma_{4}$ to the system in Equation (15), and get

$$
\left\{\begin{array}{l}
\sigma_{5} \sqrt{1-\theta_{1}^{2}} \theta_{1}=\sigma_{1}\left(-\sqrt{c\left(k_{1}\right)+\theta_{1}^{2}\left(1-\theta_{1}^{2}\right)}+\sqrt{c\left(k_{2}\right)+\theta_{1}^{2}\left(1-\theta_{1}^{2}\right)}\right) \\
\sigma_{6} \sqrt{1-\theta_{2}^{2}} \theta_{2}=\sigma_{3}\left(\sqrt{c\left(k_{1}\right)+\theta_{2}^{2}\left(1-\theta_{2}^{2}\right)}-\sqrt{c\left(k_{2}\right)+\theta_{2}^{2}\left(1-\theta_{2}^{2}\right)}\right) \\
\sigma_{2}=\sigma_{1}, \quad \sigma_{4}=\sigma_{3} .
\end{array}\right.
$$


Moreover, both $\theta_{1}^{2}\left(1-\theta_{1}^{2}\right)$ and $\theta_{2}^{2}\left(1-\theta_{2}^{2}\right)$ must be solutions $t \in[0,1 / 4]$ of the equation

$$
c\left(k_{1}\right)+c\left(k_{2}\right)+t-2 \sqrt{c\left(k_{1}\right)+t} \sqrt{c\left(k_{2}\right)+t}=0 .
$$

Such equation has the unique nonnegative solution

$$
t=t_{k_{1}, k_{2}}=\frac{1}{3}\left(2 \sqrt{c\left(k_{1}\right)^{2}-c\left(k_{1}\right) c\left(k_{2}\right)+c\left(k_{2}\right)^{2}}-c\left(k_{1}\right)-c\left(k_{2}\right)\right),
$$

which belongs to $[0,1 / 4]$ if and only if $\left(k_{1}, k_{2}\right)$ belongs to the set

$$
A=\left\{\left(k_{1}, k_{2}\right) \in\left(\frac{1}{\sqrt{2}}, 1\right)^{2}: 2 \sqrt{c\left(k_{1}\right)^{2}-c\left(k_{1}\right) c\left(k_{2}\right)+c\left(k_{2}\right)^{2}}-c\left(k_{1}\right)-c\left(k_{2}\right) \leq \frac{3}{4}\right\},
$$

i.e., as one can easily see after some computations,

$$
A=\left\{\left(k_{1}, k_{2}\right) \in \mathbb{R}: \quad k_{1} \in\left(\frac{1}{\sqrt{2}}, 1\right), \quad \frac{\sqrt{4 k_{1}^{2}-1}}{2 k_{1}} \leq k_{2} \leq \frac{1}{2 \sqrt{1-k_{1}^{2}}}, \quad k_{2}<1\right\}
$$

(the set $A$ is portrayed in Figure 4).

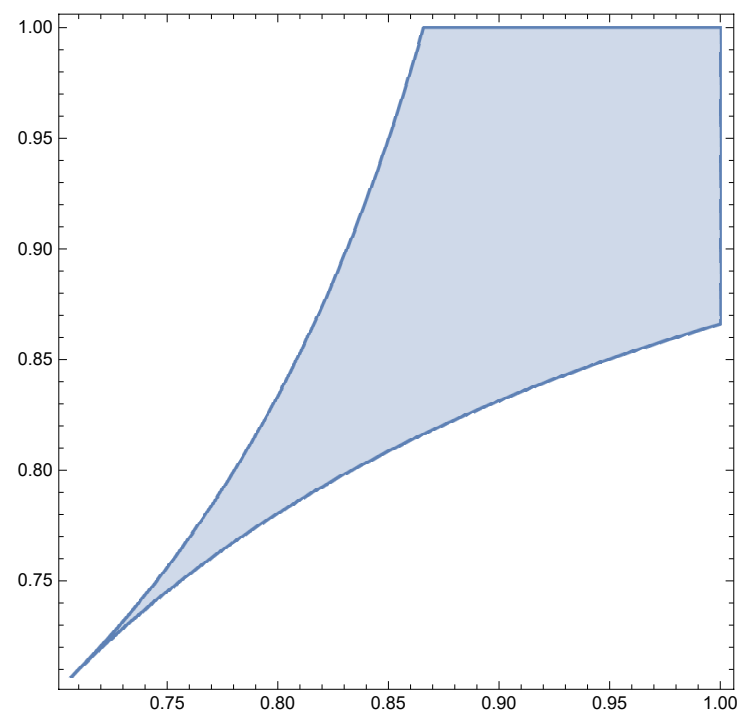

Figure 4. The set $A$. The point $(\sqrt{2} / 2, \sqrt{2} / 2)$ and the straight lines of the boundary are not included.

In this case, the equation $\theta^{2}\left(1-\theta^{2}\right)=t_{k_{1}, k_{2}}$ with $\theta \in(0,1]$ has two distinct solutions

$$
\theta_{k_{1}, k_{2}}^{ \pm}=\sqrt{\frac{1 \pm \sqrt{1-4 t_{k_{1}, k_{2}}}}{2}}
$$

if $t_{k_{1}, k_{2}} \in(0,1 / 4)$, two coincident solutions $\theta_{k_{1}, k_{2}}^{+}=\theta_{k_{1}, k_{2}}^{-}=1 / \sqrt{2}$ if $t_{k_{1}, k_{2}}=1 / 4$, and a unique solution $\theta_{k_{1}, k_{2}}^{+}=1$ if $t_{k_{1}, k_{2}}=0$ (i.e., $k_{1}=k_{2}$ ). In this latter case, we still write $\theta_{k_{1}, k_{2}}^{+}=\theta_{k_{1}, k_{2}}^{-}=1$ for future convenience. We also observe that the function $c(k)$ is positive and strictly decreasing from $(1 / \sqrt{2}, 1)$ onto $(0,+\infty)$, so that the terms within brackets on the right hand sides of the first two equations of Equation (16) have a fixed sign according as $k_{1}<k_{2}$ or $k_{1}>k_{2}$. Therefore, the system in Equation (15) turns out to be equivalent to 


$$
\left\{\begin{array}{l}
\left(k_{1}, k_{2}\right) \in A, \quad \theta_{1}, \theta_{2} \in\left\{\theta_{k_{1}, k_{2}}^{+}, \theta_{k_{1}, k_{2}}^{-}\right\} \\
\operatorname{sech}\left(\eta a_{3}\right)=\theta_{1}, \quad \operatorname{sech}\left(\eta a_{4}\right)=\theta_{2} \\
\left\{\begin{array}{l}
k_{1}<k_{2} \\
\sigma_{5}=-\sigma_{1} \\
\sigma_{6}=\sigma_{3} \\
\sigma_{2}=\sigma_{1}, \quad \sigma_{4}=\sigma_{3} .
\end{array}\right.
\end{array}\right.
$$

As a conclusion, Equation (10) is equivalent to the system in Equation (14) with the last two equations replaced by the system in Equation (19).

\section{Case $\theta_{1}=\theta_{2}, \sigma_{1}=\sigma_{3}$ and $k_{1}<k_{2}$. Proof of Theorem 1}

We focus on the case $\sigma_{1}=\sigma_{3}=1$, which gives Theorem 1, leaving the analogous case $\sigma_{1}=\sigma_{3}=-1$ to the interested reader. In such a case, condition $\left(k_{1}, k_{2}\right) \in A$ becomes

$$
\left(k_{1}, k_{2}\right) \in A^{\prime}=A \cap\left\{\left(k_{1}, k_{2}\right) \in \mathbb{R}: k_{1}<k_{2}\right\}=\left\{\left(k_{1}, k_{2}\right) \in \mathbb{R}: \quad \frac{1}{\sqrt{2}}<k_{1}<k_{2} \leq \frac{1}{2 \sqrt{1-k_{1}^{2}}}, \quad k_{2}<1\right\}
$$

and, taking into account the equivalence between Equation (15) and Equation (19), the system in Equation (14) becomes:

$$
\left\{\begin{array}{l}
\left(k_{1}, k_{2}\right) \in A^{\prime}, \quad \theta \in\left\{\theta_{k_{1}, k_{2}}^{+}, \theta_{k_{1}, k_{2}}^{-}\right\} \\
\operatorname{sech}\left(\eta a_{3}\right)=\operatorname{sech}\left(\eta a_{4}\right)=\theta, \quad a_{3}<0, \quad a_{4}>0 \\
\frac{k_{1}}{\sqrt{2 k_{1}^{2}-1}} \mathrm{cn}\left(\frac{\eta a_{1}}{\sqrt{2 k_{1}^{2}-1}} ; k_{1}\right)=\frac{k_{2}}{\sqrt{2 k_{2}^{2}-1}} \mathrm{cn}\left(\frac{\eta\left(L+a_{2}\right)}{\sqrt{2 k_{2}^{2}-1}} ; k_{2}\right)=\theta \\
\frac{k_{1}}{\sqrt{2 k_{1}^{2}-1}} \mathrm{cn}\left(\frac{\eta\left(L_{1}+a_{1}\right)}{\sqrt{2 k_{1}^{2}-1}} ; k_{1}\right)=\frac{k_{2}}{\sqrt{2 k_{2}^{2}-1}} \mathrm{cn}\left(\frac{\eta\left(L_{1}+a_{2}\right)}{\sqrt{2 k_{2}^{2}-1}} ; k_{2}\right)=\theta \\
\operatorname{sn}\left(\frac{\eta a_{1}}{\sqrt{2 k_{1}^{2}-1}} ; k_{1}\right)>0, \quad \operatorname{sn}\left(\frac{\eta\left(L_{1}+a_{1}\right)}{\sqrt{2 k_{1}^{2}-1}} ; k_{1}\right)>0 \\
\operatorname{sn}\left(\frac{\eta\left(L+a_{2}\right)}{\sqrt{2 k_{2}^{2}-1}} ; k_{2}\right)>0, \quad \operatorname{sn}\left(\frac{\eta\left(L_{1}+a_{2}\right)}{\sqrt{2 k_{2}^{2}-1}} ; k_{2}\right)>0 .
\end{array}\right.
$$

We denote by $\gamma_{j}=\gamma_{j}\left(k_{j}, \eta, \theta\right)$ the unique preimage in $\left(0, T_{j} / 4\right)$ of the value $\frac{\sqrt{2 k_{j}^{2}-1}}{k_{j}} \theta$ by the function $\mathrm{cn}\left(\frac{\eta}{\sqrt{2 k_{j}^{2}-1}}(\cdot) ; k_{j}\right)$. Then,

$$
\begin{cases}\frac{k_{1}}{\sqrt{2 k_{1}^{2}-1}} \mathrm{cn}\left(\frac{\eta a_{1}}{\sqrt{2 k_{1}^{2}-1}} ; k_{1}\right)=\theta, & \text { sn }\left(\frac{\eta a_{1}}{\sqrt{2 k_{1}^{2}-1}} ; k_{1}\right)>0 \\ \frac{k_{1}}{\sqrt{2 k_{1}^{2}-1}} \mathrm{cn}\left(\frac{\eta\left(L_{1}+a_{1}\right)}{\sqrt{2 k_{1}^{2}-1}} ; k_{1}\right)=\theta, & \text { sn }\left(\frac{\eta\left(L_{1}+a_{1}\right)}{\sqrt{2 k_{1}^{2}-1}} ; k_{1}\right)>0\end{cases}
$$

means

$$
\left\{\begin{array} { l } 
{ a _ { 1 } = \gamma _ { 1 } } \\
{ L _ { 1 } + a _ { 1 } = \gamma _ { 1 } + m T _ { 1 } \quad \text { for some } m \geq 1 , }
\end{array} \text { i.e., } \quad \left\{\begin{array}{l}
a_{1}=\gamma_{1} \\
L_{1}=m T_{1} \quad \text { for some } m \geq 1
\end{array}\right.\right.
$$


while

$$
\begin{cases}\frac{k_{2}}{\sqrt{2 k_{2}^{2}-1}} \mathrm{cn}\left(\frac{\eta\left(L+a_{2}\right)}{\sqrt{2 k_{2}^{2}-1}} ; k_{2}\right)=\theta, & \text { sn }\left(\frac{\eta\left(L+a_{2}\right)}{\sqrt{2 k_{2}^{2}-1}} ; k_{2}\right)>0 \\ \frac{k_{2}}{\sqrt{2 k_{2}^{2}-1}} \mathrm{cn}\left(\frac{\eta\left(L_{1}+a_{2}\right)}{\sqrt{2 k_{2}^{2}-1}} ; k_{2}\right)=\theta, & \text { sn }\left(\frac{\eta\left(L_{1}+a_{2}\right)}{\sqrt{2 k_{2}^{2}-1}} ; k_{2}\right)>0\end{cases}
$$

means

$$
\left\{\begin{array} { l } 
{ L + a _ { 2 } = \gamma _ { 2 } + p T _ { 2 } \text { for some } p \geq 0 } \\
{ L _ { 1 } + a _ { 2 } = \gamma _ { 2 } + q T _ { 2 } \quad \text { for some } 0 \leq q < p , }
\end{array} \text { i.e., } \quad \left\{\begin{array}{l}
L+a_{2}=\gamma_{2}+p T_{2} \text { for some } p \geq 0 \\
L_{2}=(p-q) T_{2} \text { for some } 0 \leq q<p .
\end{array}\right.\right.
$$

Hence, the system in Equation (20) becomes

$$
\left\{\begin{array}{l}
\left(k_{1}, k_{2}\right) \in A^{\prime}, \quad \theta \in\left\{\theta_{k_{1}, k_{2}}^{+}, \theta_{k_{1}, k_{2}}^{-}\right\} \\
\operatorname{sech}\left(\eta a_{3}\right)=\operatorname{sech}\left(\eta a_{4}\right)=\theta, \quad a_{3}<0, \quad a_{4}>0 \\
L_{1}=m T_{1}\left(k_{1}, \eta\right) \quad \text { for some } m \geq 1 \\
L_{2}=n T_{2}\left(k_{2}, \eta\right) \quad \text { for some } n \geq 1 \\
a_{1}=\gamma_{1}\left(k_{1}, \eta, \theta\right) \\
a_{2}=\gamma_{2}\left(k_{2}, \eta, \theta\right)+p T_{2}\left(k_{2}, \eta\right)-L \quad \text { for some } p \geq n
\end{array}\right.
$$

(observe that $\theta$ depends on both $k_{1}$ and $k_{2}$, and so do $a_{1}$ and $a_{2}$ according to the last two equations).

Remark 4. The equivalence between the systems in Equation (20) and Equation (21) does not need assumption $k_{1}<k_{2}$. On the other hand, if $k_{1}=k_{2}$, then $T_{1}\left(k_{1}, \eta\right)=T_{2}\left(k_{2}, \eta\right)$ and thus the third and fourth equations of the system in Equation (21) imply $L_{1} / L_{2} \in \mathbb{Q}$. This means that solutions to the system in Equation (10) with $k_{1}=k_{2}$ (which implies $\theta_{1}=\theta_{2}=1$ ) and $\sigma_{1}=\sigma_{3}$ cannot exist if the ratio $L_{1} / L_{2}$ is not rational.

Let us now focus on the following group of equations:

$$
\begin{cases}\left(k_{1}, k_{2}\right) \in A^{\prime} & \\ L_{1}=m T_{1}\left(k_{1}, \eta\right), & \text { for some } m \geq 1 \\ L_{2}=n T_{2}\left(k_{2}, \eta\right), & \text { for some } n \geq 1\end{cases}
$$

Recalling that $T_{j}\left(k_{j}, \eta\right)=S\left(k_{j}\right) / \eta$, this system is equivalent to

$$
\left\{\begin{array}{l}
\frac{1}{\sqrt{2}}<k_{1}<k_{2} \leq \frac{1}{2 \sqrt{1-k_{1}^{2}}}, k_{2}<1 \\
k_{1}=S^{-1}\left(\eta \frac{L_{1}}{m}\right) \quad \text { for some } m \geq 1 \\
k_{2}=S^{-1}\left(\eta \frac{L_{2}}{n}\right) \quad \text { for some } n \geq 1
\end{array}\right.
$$

and therefore, recalling that $S$ is strictly increasing and continuous from $(1 / \sqrt{2}, 1)$ onto $(0,+\infty)$, we can obtain solutions by fixing $\eta>0$ and finding $n, m \geq 1$ such that

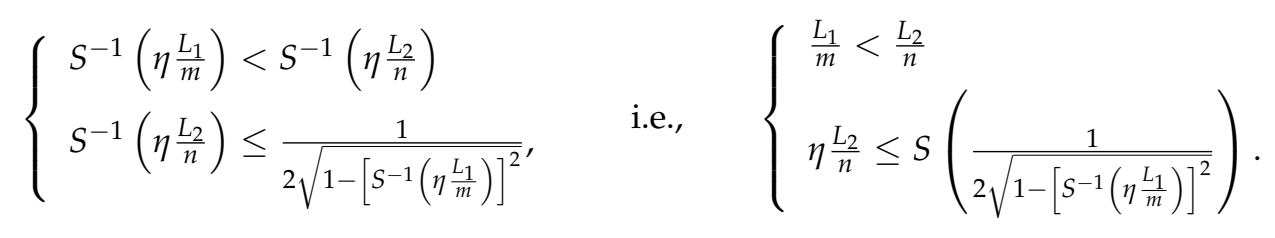


Lemma 1. One has

$$
S\left(\frac{1}{2 \sqrt{1-\left[S^{-1}(t)\right]^{2}}}\right)=t+\frac{1}{32 K_{0}^{2}} t^{3}+o\left(t^{3}\right) \quad \text { as } t \rightarrow 0^{+}
$$

(where, we recall, $K_{0}=K(1 / \sqrt{2})$ ).

Proof. We have

$$
\begin{aligned}
\lim _{t \rightarrow 0^{+}} \frac{S^{-1}(t)-\frac{1}{\sqrt{2}}-\frac{t^{2}}{32 K_{0}^{2} \sqrt{2}}}{t^{4}} & =\lim _{k \rightarrow(1 / \sqrt{2})^{+}} \frac{S^{-1}(S(k))-\frac{1}{\sqrt{2}}-\frac{S(k)^{2}}{32 K_{0}^{2} \sqrt{2}}}{S(k)^{4}} \\
& =\lim _{k \rightarrow(1 / \sqrt{2})^{+}} \frac{k-\frac{1}{\sqrt{2}}-\frac{16 K(k)^{2}\left(2 k^{2}-1\right)}{32 K_{0}^{2} \sqrt{2}}}{2^{8} K(k)^{4}\left(2 k^{2}-1\right)^{2}} \\
& =\frac{1}{2^{10} K_{0}^{2}} \lim _{k \rightarrow(1 / \sqrt{2})^{+}} \frac{2 K_{0}^{2}-K(k)^{2}(\sqrt{2} k+1)}{K(k)^{4}(\sqrt{2} k+1)^{2}(k-1 / \sqrt{2})}
\end{aligned}
$$

where, setting $K_{0}^{\prime}=K^{\prime}(1 / \sqrt{2})$, by De L'Hôpital's rule, we get

$$
\lim _{k \rightarrow(1 / \sqrt{2})^{+}} \frac{2 K_{0}^{2}-K(k)^{2}(\sqrt{2} k+1)}{k-1 / \sqrt{2}}=-4 K_{0} K_{0}^{\prime}-K_{0}^{2} \sqrt{2} .
$$

Hence, we conclude

$$
\lim _{t \rightarrow 0^{+}} \frac{S^{-1}(t)-\frac{1}{\sqrt{2}}-\frac{t^{2}}{32 K_{0}^{2} \sqrt{2}}}{t^{4}}=-\frac{K_{0}+2 \sqrt{2} K_{0}^{\prime}}{2^{11} \sqrt{2} K_{0}^{5}}
$$

i.e.,

$$
S^{-1}(t)=\frac{1}{\sqrt{2}}+c_{1} t^{2}-c_{2} t^{4}+o\left(t^{4}\right) \quad \text { as } t \rightarrow 0^{+}
$$

where $c_{1}=\frac{1}{32 \sqrt{2} K_{0}^{2}}$ and $c_{2}=\frac{K_{0}+2 \sqrt{2} K_{0}^{\prime}}{2^{11} \sqrt{2} K_{0}^{5}}$. This implies

$$
\begin{aligned}
\frac{1}{2 \sqrt{1-S^{-1}(t)^{2}}} & =\frac{1}{2 \sqrt{\frac{1}{2}-\frac{2}{\sqrt{2}} c_{1} t^{2}-\left(c_{1}^{2}-\sqrt{2} c_{2}\right) t^{4}+o\left(t^{4}\right)}} \\
& =\frac{1}{\sqrt{2} \sqrt{1-2 \sqrt{2} c_{1} t^{2}-2\left(c_{1}^{2}-\sqrt{2} c_{2}\right) t^{4}+o\left(t^{4}\right)}} \\
& =\frac{1}{\sqrt{2}}+c_{1} t^{2}+\left(2 \sqrt{2} c_{1}^{2}-c_{2}\right) t^{4}+o\left(t^{4}\right)
\end{aligned}
$$

Using De L'Hôpital's rule again, we now compute

$$
\lim _{k \rightarrow(1 / \sqrt{2})^{+}} \frac{S(k)-2^{11 / 4} K_{0}(k-1 / \sqrt{2})^{1 / 2}}{(k-1 / \sqrt{2})^{3 / 2}}=\lim _{k \rightarrow(1 / \sqrt{2})^{+}} \frac{S^{\prime}(k)-2^{7 / 4} K_{0}(k-1 / \sqrt{2})^{-1 / 2}}{\frac{3}{2}(k-1 / \sqrt{2})^{1 / 2}}
$$




$$
\begin{aligned}
& =\frac{2}{3} \lim _{k \rightarrow(1 / \sqrt{2})^{+}} \frac{\frac{8 k}{\sqrt{2 k^{2}-1}} K(k)+4 \sqrt{2 k^{2}-1} K^{\prime}(k)-\frac{2^{7 / 4} K_{0}}{(k-1 / \sqrt{2})^{1 / 2}}}{(k-1 / \sqrt{2})^{1 / 2}} \\
& =\frac{2^{15 / 4}}{3} K_{0}^{\prime}+\frac{2}{3} \lim _{k \rightarrow(1 / \sqrt{2})^{+}} \frac{\frac{8 k K(k)}{\sqrt[4]{2} \sqrt{\sqrt{2} k+1}}-2^{7 / 4} K_{0}}{k-1 / \sqrt{2}} \\
& =\frac{2^{15 / 4}}{3} K_{0}^{\prime}+\frac{2}{3} \lim _{k \rightarrow(1 / \sqrt{2})^{+}} \frac{\frac{8 k\left(K(k)-K_{0}\right)}{\sqrt[4]{2} \sqrt{\sqrt{2} k+1}}+\left(\frac{8 k}{\sqrt[4]{2} \sqrt{\sqrt{2} k+1}}-2^{7 / 4}\right) K_{0}}{k-1 / \sqrt{2}}=2^{5 / 4} K_{0}+2^{11 / 4} K_{0}^{\prime}
\end{aligned}
$$

where the result follows because $K(k)-K_{0} \sim K_{0}^{\prime}(k-1 / \sqrt{2})$ as $k \rightarrow(1 / \sqrt{2})^{+}$and

$$
\begin{aligned}
\frac{8 k}{\sqrt[4]{2} \sqrt{\sqrt{2} k+1}}-2^{7 / 4} & =2^{7 / 4} \frac{2 k-\sqrt{\sqrt{2} k+1}}{\sqrt{\sqrt{2} k+1}}=2^{7 / 4} \frac{4 k^{2}-\sqrt{2} k-1}{\sqrt{\sqrt{2} k+1}(2 k+\sqrt{\sqrt{2} k+1})} \\
& =2^{7 / 4} \frac{(4 k+\sqrt{2})(k-1 / \sqrt{2})}{\sqrt{\sqrt{2} k+1}(2 k+\sqrt{\sqrt{2} k+1})} .
\end{aligned}
$$

This means

$$
S(k)=2^{11 / 4} K_{0}(k-1 / \sqrt{2})^{1 / 2}+\left(2^{5 / 4} K_{0}+2^{11 / 4} K_{0}^{\prime}\right)(k-1 / \sqrt{2})^{3 / 2}+o\left((k-1 / \sqrt{2})^{3 / 2}\right)
$$

as $k \rightarrow(1 / \sqrt{2})^{+}$and therefore we deduce that as $t \rightarrow 0^{+}$one has (note that $2^{11 / 4} K_{0} \sqrt{c_{1}}=1$ )

$$
\begin{aligned}
S\left(\frac{1}{\left.2 \sqrt{1-S^{-1}(t)^{2}}\right)=}\right. & 2^{11 / 4} K_{0} \sqrt{c_{1}} t\left(1+\frac{2 \sqrt{2} c_{1}^{2}-c_{2}}{c_{1}} t^{2}+o\left(t^{2}\right)\right)^{1 / 2}+ \\
& +\left(2^{5 / 4} K_{0}+2^{11 / 4} K_{0}^{\prime}\right) c_{1} \sqrt{c_{1}} t^{3}\left(1+\frac{2 \sqrt{2} c_{1}^{2}-c_{2}}{c_{1}} t^{2}+o\left(t^{2}\right)\right)^{3 / 2}+o\left(t^{3}\right) \\
= & t\left(1+\frac{1}{2} \frac{2 \sqrt{2} c_{1}^{2}-c_{2}}{c_{1}} t^{2}+o\left(t^{2}\right)\right)+ \\
& +\left(2^{5 / 4} K_{0}+2^{11 / 4} K_{0}^{\prime}\right) c_{1} \sqrt{c_{1}} t^{3}\left(1+\frac{3}{2} \frac{2 \sqrt{2} c_{1}^{2}-c_{2}}{c_{1}} t^{2}+o\left(t^{2}\right)\right)+o\left(t^{3}\right) \\
= & t+\left(2^{11 / 4} K_{0} \sqrt{c_{1}} \frac{1}{2} \frac{2 \sqrt{2} c_{1}^{2}-c_{2}}{c_{1}}+\left(2^{5 / 4} K_{0}+2^{11 / 4} K_{0}^{\prime}\right) c_{1} \sqrt{c_{1}}\right) t^{3}+o\left(t^{3}\right) .
\end{aligned}
$$

Simplifying the coefficient of $t^{3}$, this gives the result.

Thanks to Lemma 1, the system in Equation (24) becomes

$$
0<\frac{m}{n}-\frac{L_{1}}{L_{2}} \leq \frac{L_{1}^{3} \eta^{2}}{32 K_{0}^{2} L_{2}} \frac{1}{m^{2}}+\zeta_{m}
$$

where $\left(\zeta_{m}\right)_{m}$ is a suitable sequence (also dependent on $\left.L_{1}, L_{2}, \eta\right)$ such that $\zeta_{m}=o\left(m^{-2}\right)$ as $m \rightarrow \infty$. Notice that, according to systems (23) and (24), the equality sign in the second inequality amounts to $k_{2}=\frac{1}{2 \sqrt{1-k_{1}^{2}}}$. 
Proof of Theorem 1. Since $L_{1} / L_{2} \in \mathbb{R} \backslash \mathbb{Q}$, by ([21], Corollary 1.9) there exist infinitely many rational numbers $m / n$ such that

$$
0<\frac{m}{n}-\frac{L_{1}}{L_{2}}<\frac{1}{n^{2}}
$$

This implies $n L_{1} / L_{2}<m<n L_{1} / L_{2}+1$ and thus $m=\left[n L_{1} / L_{2}+1\right]$. Since the denominators of such rationals $m / n$ must be infinite, we may arrange them in a diverging sequence $\left(n_{h}\right) \subset \mathbb{N}$; accordingly, the corresponding numerators are $m_{h}=\left[n_{h} L_{1} / L_{2}+1\right]$. Now, let $\eta>4 \sqrt{2} K_{0}\left(L_{1} L_{2}\right)^{-1 / 2}$ and fix $\varepsilon>0$ such that

$$
\eta^{2}>\left(\frac{L_{1}}{L_{2}}+\varepsilon\right)^{2} \frac{32 K_{0}^{2} L_{2}}{L_{1}^{3}}
$$

Since Equation (28) implies that $m_{h} / n_{h} \rightarrow L_{1} / L_{2}$ as $h \rightarrow \infty$, for $h$ large enough, we have that $m_{h} / n_{h}<L_{1} / L_{2}+\varepsilon$, so that

$$
\frac{1}{n_{h}^{2}}<\left(\frac{L_{1}}{L_{2}}+\varepsilon\right)^{2} \frac{1}{m_{h}^{2}}<\frac{L_{1}^{3} \eta^{2}}{32 K_{0}^{2} L_{2}} \frac{1}{m_{h}^{2}}
$$

Hence, up to further enlarging $h$, Equation (28) gives

$$
0<\frac{m_{h}}{n_{h}}-\frac{L_{1}}{L_{2}}<\left(\frac{L_{1}}{L_{2}}+\varepsilon\right)^{2} \frac{1}{m_{h}^{2}}<\frac{L_{1}^{3} \eta^{2}}{32 K_{0}^{2} L_{2}} \frac{1}{m_{h}^{2}}+\zeta_{m_{h}}
$$

so that $n_{h}$ and $m_{h}$ satisfy Equation (27). For every $h$, this provides solutions to the system in Equation (22) by taking $k_{1}=k_{1, h}=S^{-1}\left(\eta L_{1} / m_{h}\right)$ and $k_{2}=k_{2, h}=S^{-1}\left(\eta L_{2} / n_{h}\right)$, and thus solutions to the system in Equation (21) by choosing $\theta=\theta_{h} \in\left\{\theta_{k_{1, h}, k_{2, h}}^{+}, \theta_{k_{1, h}, k_{2, h}}^{-}\right\}$, taking $p$ as the unique integer such that

$$
0 \leq \gamma_{2}\left(k_{2, h}, \eta, \theta_{h}\right)+p T_{2}\left(k_{2, h}, \eta\right)-L<T_{2}\left(k_{2, h}, \eta\right)
$$

(where $\left.T_{2}\left(k_{2, h}, \eta\right)=L_{2} / n_{h}\right)$, which turns out to be greater than or equal to $n_{h}$, and defining $a_{1}, a_{2}, a_{3}, a_{4}$ according to the second, fifth and sixth equation of the system. Note that $\theta_{k_{1, h}, k_{2, h}}^{+}$and $\theta_{k_{1, h}, k_{2, h}}^{-}$are different for all $h$, since $t_{k_{1, h}, k_{2, h}} \neq 0$ (because $k_{1, h} \neq k_{2, h}$ ) and $t_{k_{1, h}, k_{2, h}} \neq 1 / 4$ (because of the strict inequality signs in Equation (29)). Up to discarding a finite number of terms of the sequence $\left(n_{h}\right)$, the proof is complete.

4. Case $\theta_{1}=\theta_{2}, \sigma_{1}=\sigma_{3}$ and $k_{1}>k_{2}$. Proof of Theorem 2

As in the previous section, we focus on the case $\sigma_{1}=\sigma_{3}=1$. In this case, the system in Equation (14) becomes again the system in Equation (21), but with $\left(k_{1}, k_{2}\right) \in A^{\prime}$ replaced by $\left(k_{1}, k_{2}\right) \in A^{\prime \prime}$, where

$$
A^{\prime \prime}=A \cap\left\{\left(k_{1}, k_{2}\right) \in \mathbb{R}: k_{1}>k_{2}\right\}=\left\{\left(k_{1}, k_{2}\right) \in \mathbb{R}: \frac{\sqrt{4 k_{1}^{2}-1}}{2 k_{1}} \leq k_{2}<k_{1}<1\right\} .
$$

Then, Equation (22) is now equivalent to the system

$$
\left\{\begin{array}{l}
\sqrt{1-\frac{1}{4 k_{1}^{2}}} \leq k_{2}<k_{1}<1 \\
k_{1}=S^{-1}\left(\eta \frac{L_{1}}{m}\right) \quad \text { for some } m \geq 1 \\
k_{2}=S^{-1}\left(\eta \frac{L_{2}}{n}\right) \quad \text { for some } n \geq 1,
\end{array}\right.
$$


i.e.,

$$
\left\{\begin{array}{l}
\frac{L_{2}}{n}<\frac{L_{1}}{m} \\
\eta \frac{L_{2}}{n} \geq S\left(\sqrt{1-\frac{1}{4 S^{-1}\left(\eta \frac{L_{1}}{m}\right)^{2}}}\right) \\
k_{1}=S^{-1}\left(\eta \frac{L_{1}}{m}\right), \quad k_{2}=S^{-1}\left(\eta \frac{L_{2}}{n}\right)
\end{array}\right.
$$

with $\eta>0$ and $n, m \in \mathbb{N}$.

Lemma 2. One has

$$
S\left(\sqrt{1-\frac{1}{4 S^{-1}(t)^{2}}}\right)=t-\frac{1}{32 K_{0}^{2}} t^{3}+o\left(t^{3}\right) \quad \text { as } t \rightarrow 0^{+}
$$

(where, we recall, $K_{0}=K(1 / \sqrt{2})$ ).

Proof. Since $S^{-1}(t)=\frac{1}{\sqrt{2}}+c_{1} t^{2}-c_{2} t^{4}+o\left(t^{4}\right)$ as $t \rightarrow 0^{+}$(see Equation (25)), we have

$$
\begin{aligned}
1-\frac{1}{2 S^{-1}(t)^{2}} & =1-\frac{1}{2\left(S^{-1}(t)-1 / \sqrt{2}+1 / \sqrt{2}\right)^{2}} \\
& =1-\frac{1}{2} \frac{1}{\left(S^{-1}(t)-1 / \sqrt{2}\right)^{2}+1 / 2+2\left(S^{-1}(t)-1 / \sqrt{2}\right) / \sqrt{2}} \\
& =1-\frac{1}{2} \frac{1}{\left(c_{1} t^{2}-c_{2} t^{4}+o\left(t^{4}\right)\right)^{2}+1 / 2+2\left(c_{1} t^{2}-c_{2} t^{4}+o\left(t^{4}\right)\right) / \sqrt{2}} \\
& =1-\frac{1}{1+2 c_{1} \sqrt{2} t^{2}+2\left(c_{1}^{2}-c_{2} \sqrt{2}\right) t^{4}+o\left(t^{4}\right)} \\
& =2 c_{1} \sqrt{2} t^{2}-2\left(3 c_{1}^{2}+c_{2} \sqrt{2}\right) t^{4}+o\left(t^{4}\right)
\end{aligned}
$$

and therefore

$$
\begin{aligned}
\sqrt{1-\frac{1}{4 S^{-1}(t)^{2}}} & =\frac{1}{\sqrt{2}} \sqrt{1+\left(1-\frac{1}{2 S^{-1}(t)^{2}}\right)} \\
& =\frac{1}{\sqrt{2}}\left(1+\frac{1}{2}\left(1-\frac{1}{2 S^{-1}(t)^{2}}\right)-\frac{1}{8}\left(1-\frac{1}{2 S^{-1}(t)^{2}}\right)^{2}+o\left(\left(1-\frac{1}{2 S^{-1}(t)^{2}}\right)^{2}\right)\right) \\
& =\frac{1}{\sqrt{2}}+c_{1} t^{2}-\left(2 \sqrt{2} c_{1}^{2}+c_{2}\right) t^{4}+o\left(t^{4}\right) .
\end{aligned}
$$


Hence, using the expansion in Equation (25), we deduce that

$$
\begin{aligned}
S\left(\sqrt{\left.1-\frac{1}{4 S^{-1}(t)^{2}}\right)=}\right. & 2^{11 / 4} K_{0} \sqrt{c_{1}} t\left(1-\frac{2 \sqrt{2} c_{1}^{2}+c_{2}}{c_{1}} t^{2}+o\left(t^{2}\right)\right)^{1 / 2}+ \\
& +\left(2^{5 / 4} K_{0}+2^{11 / 4} K_{0}^{\prime}\right) c_{1} \sqrt{c_{1}} t^{3}\left(1-\frac{2 \sqrt{2} c_{1}^{2}+c_{2}}{c_{1}} t^{2}+o\left(t^{2}\right)\right)^{3 / 2}+o\left(t^{3}\right) \\
= & t\left(1-\frac{1}{2} \frac{2 \sqrt{2} c_{1}^{2}+c_{2}}{c_{1}} t^{2}+o\left(t^{2}\right)\right)+ \\
& +\left(2^{5 / 4} K_{0}+2^{11 / 4} K_{0}^{\prime}\right) c_{1} \sqrt{c_{1}} t^{3}\left(1-\frac{3}{2} \frac{2 \sqrt{2} c_{1}^{2}+c_{2}}{c_{1}} t^{2}+o\left(t^{2}\right)\right)+o\left(t^{3}\right) \\
= & t+\left(\left(2^{5 / 4} K_{0}+2^{11 / 4} K_{0}^{\prime}\right) c_{1} \sqrt{c_{1}}-2^{11 / 4} K_{0} \sqrt{c_{1}} \frac{1}{2} \frac{2 \sqrt{2} c_{1}^{2}+c_{2}}{c_{1}}\right) t^{3}+o\left(t^{3}\right) .
\end{aligned}
$$

Simplifying the coefficient of $t^{3}$, the result ensues.

By Lemma 2, the first two conditions of the system in Equation (24) become

$$
0>\frac{m}{n}-\frac{L_{1}}{L_{2}} \geq-\frac{L_{1}^{3} \eta^{2}}{32 K_{0}^{2} L_{2}} \frac{1}{m^{2}}+\zeta_{m}
$$

where $\left(\zeta_{m}\right)_{m}$ is a suitable sequence such that $\zeta_{m}=o\left(m^{-2}\right)$ as $m \rightarrow \infty$. Notice that the equality sign in the second inequality amounts to $k_{2}=\frac{\sqrt{4 k_{1}^{2}-1}}{2 k_{1}}$.

Proof of Theorem 2. Since $L_{1} / L_{2} \in \mathbb{R} \backslash \mathbb{Q}$, by ([21], Corollary 1.9) there exist infinitely many rational numbers $m / n$ such that

$$
0>\frac{m}{n}-\frac{L_{1}}{L_{2}}>-\frac{1}{n^{2}} .
$$

This implies $n L_{1} / L_{2}-1<m<n L_{1} / L_{2}$ and thus $m=\left[n L_{1} / L_{2}\right]$. Proceeding exactly as in the proof of Theorem 1 , the result follows.

\section{Case $\theta_{1}=\theta_{2}$ and $\sigma_{1}=-\sigma_{3}$. Proof of Theorem 3}

We focus on the case $\theta_{1}=\theta_{2}=\theta_{k_{1}, k_{2}}^{+}$and $\sigma_{1}=-\sigma_{3}=1$, which gives Theorem 3, leaving the analogous cases $\theta_{1}=\theta_{2}=\theta_{k_{1}, k_{2}}^{-}$or $\sigma_{1}=-\sigma_{3}=-1$ to the interested reader. In such a case, the system in Equation (14) becomes

$$
\left\{\begin{array}{l}
\left(k_{1}, k_{2}\right) \in A \\
\frac{k_{1}}{\sqrt{2 k_{1}^{2}-1}} \mathrm{cn}\left(\frac{\eta a_{1}}{\sqrt{2 k_{1}^{2}-1}} ; k_{1}\right)=\frac{k_{2}}{\sqrt{2 k_{2}^{2}-1}} \mathrm{cn}\left(\frac{\eta\left(L+a_{2}\right)}{\sqrt{2 k_{2}^{2}-1}} ; k_{2}\right)=\operatorname{sech}\left(\eta a_{3}\right)=\theta_{k_{1}, k_{2}}^{+} \\
\frac{k_{1}}{\sqrt{2 k_{1}^{2}-1}} \mathrm{cn}\left(\frac{\eta\left(L_{1}+a_{1}\right)}{\sqrt{2 k_{1}^{2}-1}} ; k_{1}\right)=\frac{k_{2}}{\sqrt{2 k_{2}^{2}-1}} \mathrm{cn}\left(\frac{\eta\left(L_{1}+a_{2}\right)}{\sqrt{2 k_{2}^{2}-1}} ; k_{2}\right)=\operatorname{sech}\left(\eta a_{4}\right)=\theta_{k_{1}, k_{2}}^{+} \\
\sigma_{2}=-\sigma_{4}=1 \\
\left\{\begin{array} { l } 
{ k _ { 1 } < k _ { 2 } } \\
{ \sigma _ { 5 } = \sigma _ { 6 } = - 1 }
\end{array} \vee \left\{\begin{array} { l } 
{ k _ { 1 } > k _ { 2 } } \\
{ \sigma _ { 5 } = \sigma _ { 6 } = 1 }
\end{array} \vee \left\{\begin{array}{l}
k_{1}=k_{2} \\
a_{3}=a_{4}=0
\end{array}\right.\right.\right.
\end{array}\right.
$$


that is

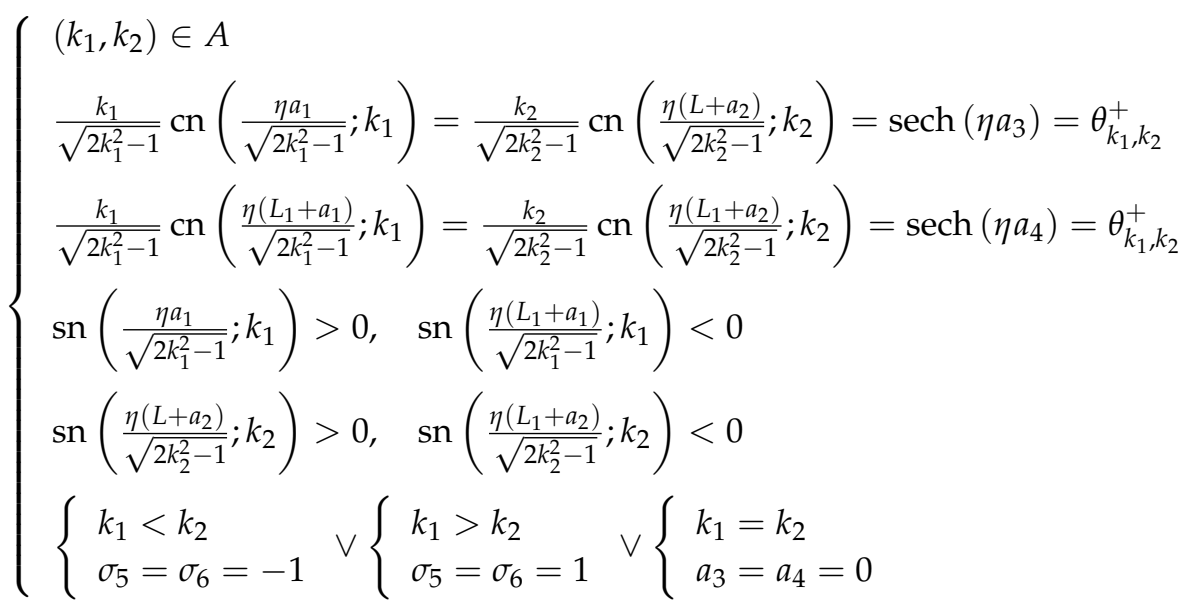

Defining $\gamma_{j}\left(k_{j}, \eta, \theta\right)$ as in Section 3, we have that

$$
\left\{\begin{array}{l}
\frac{k_{1}}{\sqrt{2 k_{1}^{2}-1}} \mathrm{cn}\left(\frac{\eta a_{1}}{\sqrt{2 k_{1}^{2}-1}} ; k_{1}\right)=\theta_{k_{1}, k_{2}}^{+}, \quad \text { sn }\left(\frac{\eta a_{1}}{\sqrt{2 k_{1}^{2}-1}} ; k_{1}\right)>0 \\
\frac{k_{1}}{\sqrt{2 k_{1}^{2}-1}} \mathrm{cn}\left(\frac{\eta\left(L_{1}+a_{1}\right)}{\sqrt{2 k_{1}^{2}-1}} ; k_{1}\right)=\theta_{k_{1}, k_{2}}^{+}, \quad \text { sn }\left(\frac{\eta\left(L_{1}+a_{1}\right)}{\sqrt{2 k_{1}^{2}-1}} ; k_{1}\right)<0
\end{array}\right.
$$

means

$$
\left\{\begin{array}{l}
a_{1}=\gamma_{1}\left(k_{1}, \eta, \theta_{k_{1}, k_{2}}^{+}\right) \\
L_{1}=m T_{1}\left(k_{1}, \eta\right)-2 \gamma_{1}\left(k_{1}, \eta, \theta_{k_{1}, k_{2}}^{+}\right) \quad \text { for some } m \geq 1
\end{array}\right.
$$

and

$$
\left\{\begin{array}{l}
\frac{k_{2}}{\sqrt{2 k_{2}^{2}-1}} \mathrm{cn}\left(\frac{\eta\left(L+a_{2}\right)}{\sqrt{2 k_{2}^{2}-1}} ; k_{2}\right)=\theta_{k_{1}, k_{2}}^{+}, \quad \text { sn }\left(\frac{\eta\left(L+a_{2}\right)}{\sqrt{2 k_{2}^{2}-1}} ; k_{2}\right)>0 \\
\frac{k_{2}}{\sqrt{2 k_{2}^{2}-1}} \mathrm{cn}\left(\frac{\eta\left(L_{1}+a_{2}\right)}{\sqrt{2 k_{2}^{2}-1}} ; k_{2}\right)=\theta_{k_{1}, k_{2}}^{+}, \quad \text { sn }\left(\frac{\eta\left(L_{1}+a_{2}\right)}{\sqrt{2 k_{2}^{2}-1}} ; k_{2}\right)<0
\end{array}\right.
$$

means

$$
\left\{\begin{array}{l}
L_{2}=(n-m) T_{2}\left(k_{2}, \eta\right)+2 \gamma_{2}\left(k_{2}, \eta, \theta_{k_{1}, k_{2}}^{+}\right) \quad \text { for some } n \geq m \\
a_{2}=\gamma_{2}\left(k_{2}, \eta, \theta_{k_{1}, k_{2}}^{+}\right)-L+p T_{2}\left(k_{2}, \eta\right) \quad \text { for some } p \geq n-m+1
\end{array}\right.
$$

where $m$ is the same integer of the system in Equation (32). Hence, the system in Equation (31) amounts to

$$
\left\{\begin{array}{l}
\left(k_{1}, k_{2}\right) \in A \\
L_{1}=m T_{1}\left(k_{1}, \eta\right)-2 \gamma_{1}\left(k_{1}, \eta, \theta_{k_{1}, k_{2}}^{+}\right) \quad \text { for some } m \geq 1 \\
L_{2}=(n-m) T_{2}\left(k_{2}, \eta\right)+2 \gamma_{2}\left(k_{2}, \eta, \theta_{k_{1}, k_{2}}^{+}\right) \quad \text { for some } n \geq m \\
a_{1}=\gamma_{1}\left(k_{1}, \eta, \theta_{k_{1}, k_{2}}^{+}\right) \\
a_{2}=\gamma_{2}\left(k_{2}, \eta, \theta_{k_{1}, k_{2}}^{+}\right)-L+p T_{2}\left(k_{2}, \eta\right) \quad \text { for some } p \geq n-m+1 \\
\operatorname{sech}\left(\eta a_{3}\right)=\operatorname{sech}\left(\eta a_{4}\right)=\theta_{k_{1}, k_{2}}^{+} \\
\left\{\begin{array} { l } 
{ k _ { 1 } < k _ { 2 } } \\
{ a _ { 3 } , a _ { 4 } < 0 }
\end{array} \vee \left\{\begin{array} { l } 
{ k _ { 1 } > k _ { 2 } } \\
{ a _ { 3 } , a _ { 4 } > 0 }
\end{array} \vee \left\{\begin{array}{l}
k_{1}=k_{2} \\
a_{3}=a_{4}=0 .
\end{array}\right.\right.\right.
\end{array}\right.
$$

Remark 5. Suppose $L_{1} / L_{2} \notin \mathbb{Q}$. If we assume $k_{1}=k_{2}$ in the system in Equation (14), then we have $\theta_{1}=\theta_{2}=1$ and $\sigma_{1}=-\sigma_{3}$ (see Remark 4). Hence, a solution to the problem in. Equation (6) with plus 
sign gives rise to a solution to the system in Equation (33). On the other hand, a solution to the system in Equation (33) with $k_{1}=k_{2}$ is such that $L=L_{1}+L_{2}=n T$ and $a_{2}=a_{1}-L+p T=a_{1}+(p-n) T$, where $T=T_{1}\left(k_{1}, \eta\right)=T_{2}\left(k_{2}, \eta\right), a_{1} \in(0, T / 4)$ and $a_{2} \in[0, T)$. This forces $p=n$ and thus $a_{1}=a_{2}$, so that the corresponding solution to the problem in Equation (6) is periodic on the circle.

Now, recall that $T_{j}\left(k_{j}, \eta\right):=\frac{S\left(k_{j}\right)}{\eta}$. By the definition of $\gamma_{j}=\gamma_{j}\left(k_{j}, \eta, \theta_{k_{1}, k_{2}}^{+}\right)$, one has

$$
\text { cn }\left(\frac{\eta}{\sqrt{2 k_{j}^{2}-1}} \gamma_{j} ; k_{j}\right)=\frac{\sqrt{2 k_{j}^{2}-1}}{k_{j}} \theta_{k_{1}, k_{2}}^{+}
$$

with $\gamma_{j} \in\left(0, T_{j} / 4\right)$. This implies

$$
0<\frac{\eta}{\sqrt{2 k_{j}^{2}-1}} \gamma_{j}<\frac{\eta}{\sqrt{2 k_{j}^{2}-1}} \frac{S\left(k_{j}\right)}{4 \eta}=\frac{S\left(k_{j}\right)}{4 \sqrt{2 k_{j}^{2}-1}}=K\left(k_{j}\right)
$$

and therefore Equation (34) yields that

$$
\gamma_{j}\left(k_{j}, \eta, \theta_{k_{1}, k_{2}}^{+}\right)=\frac{\sqrt{2 k_{j}^{2}-1}}{\eta} \operatorname{arccn}\left(\frac{\sqrt{2 k_{j}^{2}-1}}{k_{j}} \theta_{k_{1}, k_{2}}^{+} ; k_{j}\right) .
$$

Hence, defining

$$
\gamma\left(k_{1}, k_{2}\right):=\sqrt{2 k_{1}^{2}-1} \operatorname{arccn}\left(\frac{\sqrt{2 k_{1}^{2}-1}}{k_{1}} \theta_{k_{1}, k_{2}}^{+} ; k_{1}\right)=\sqrt{2 k_{1}^{2}-1} \int_{\frac{\sqrt{2 k_{1}^{2}-1}}{k_{1}} \theta_{k_{1}, k_{2}}^{+}}^{1} \frac{d t}{\sqrt{\left(1-t^{2}\right)\left(1-k_{1}^{2}\left(1-t^{2}\right)\right)}}
$$

and observing that $\theta_{k_{1}, k_{2}}^{+}=\theta_{k_{2}, k_{1}}^{+}$one has

$$
\gamma_{1}\left(k_{1}, \eta, \theta_{k_{1}, k_{2}}^{+}\right)=\frac{1}{\eta} \gamma\left(k_{1}, k_{2}\right) \quad \text { and } \quad \gamma_{2}\left(k_{2}, \eta, \theta_{k_{1}, k_{2}}^{+}\right)=\frac{1}{\eta} \gamma\left(k_{2}, k_{1}\right) .
$$

Thus, the first three equations of the system in Equation (33) are equivalent to

$$
\left\{\begin{array}{l}
\left(k_{1}, k_{2}\right) \in A \\
\eta L_{1}=m S\left(k_{1}\right)-2 \gamma\left(k_{1}, k_{2}\right) \quad \text { for some } m \geq 1 \\
\eta L_{2}=(n-m) S\left(k_{2}\right)+2 \gamma\left(k_{2}, k_{1}\right) \quad \text { for some } n \geq m
\end{array}\right.
$$

To prove Theorem 3, we use the following lemma, concerning the existence of a globally defined implicit function. Its proof is classical, so we leave it to the interested reader.

Lemma 3. Let $b_{i} \in \mathbb{R}$ for $i=1, \ldots, 4$ and let $G:\left(b_{1}, b_{2}\right) \times\left(b_{3}, b_{4}\right) \rightarrow \mathbb{R}$ be a continuous function such that for all $x \in\left(b_{1}, b_{2}\right)$ the following properties hold:

- the mapping $G(x, \cdot)$ is strictly increasing on $\left(b_{3}, b_{4}\right)$;

- $\lim _{y \rightarrow b_{3}^{+}} G(x, y)<0$ and $\lim _{y \rightarrow b_{4}^{-}} G(x, y)>0$.

Then, the set of solutions to the equation $G(x, y)=0$ is the graph of a continuous function $g:\left(b_{1}, b_{2}\right) \rightarrow$ $\left(b_{3}, b_{4}\right)$. 
Proof of Theorem 3. Let $n>m \geq 1$ and for $\left(k_{1}, k_{2}\right) \in A$ define the continuous functions

$$
F_{m}\left(k_{1}, k_{2}\right):=m S\left(k_{1}\right)-2 \gamma\left(k_{1}, k_{2}\right) \quad \text { and } \quad F_{m, n}\left(k_{1}, k_{2}\right):=(n-m) S\left(k_{2}\right)+2 \gamma\left(k_{2}, k_{1}\right) .
$$

We also define $F_{m}$ and $F_{m, n}$ on the segments $\left\{\left(k_{1}, 1\right): \sqrt{3} / 2 \leq k_{1}<1\right\}$ and $\left\{\left(1, k_{2}\right): \sqrt{3} / 2 \leq k_{2}<1\right\}$ of the boundary of $A$, respectively, where the above definitions also make sense.

Fix $\sqrt{3} / 2<\lambda<1$ such that the square $Q=[\lambda, 1] \times[\lambda, 1]$ is contained into the closure of $A$ and the partial derivatives $\partial F_{1} / \partial k_{1}$ and $\partial F_{1,2} / \partial k_{2}$ are strictly positive on $Q$. The existence of such a square can be checked by using the explicit expressions

$$
\begin{aligned}
F_{1}\left(k_{1}, k_{2}\right) & =2 \sqrt{2 k_{1}^{2}-1}\left(2 K\left(k_{1}\right)-\int_{\frac{\sqrt{2 k_{1}^{2}-1}}{k_{1}} \theta_{k_{1}, k_{2}}^{+}}^{1} \frac{d t}{\sqrt{\left(1-t^{2}\right)\left(1-k_{1}^{2}\left(1-t^{2}\right)\right)}}\right), \\
F_{1,2}\left(k_{1}, k_{2}\right) & =2 \sqrt{2 k_{2}^{2}-1}\left(2 K\left(k_{2}\right)+\int_{\frac{\sqrt{2 k_{2}^{2}-1}}{k_{2}} \theta_{k_{1}, k_{2}}^{+}}^{1} \frac{d t}{\sqrt{\left(1-t^{2}\right)\left(1-k_{2}^{2}\left(1-t^{2}\right)\right)}}\right),
\end{aligned}
$$

where $\theta_{k_{1}, k_{2}}^{+}$is given by Equation (18). Similarly, one checks that also $F_{1}$ is strictly positive on $Q$, while $F_{1,2}$ obviously is. Consequently, $\partial F_{m} / \partial k_{1}, \partial F_{m, n} / \partial k_{2}, F_{m}$ and $F_{m, n}$ are also strictly positive on $Q$ (recall that the function $S$ is strictly increasing and positive). Define

$$
\mu_{m}:=\max _{\lambda \leq k_{2} \leq 1} F_{m}\left(\lambda, k_{2}\right), \quad \mu_{m, n}:=\max _{\lambda \leq k_{1} \leq 1} F_{m, n}\left(k_{1}, \lambda\right) \quad \text { and } \quad \eta_{m, n}:=\frac{\max \left\{\mu_{m}, \mu_{m, n}\right\}}{L_{1}},
$$

and let $\eta>\eta_{m, n}$, so that $\eta L_{2}>\eta L_{1}>\max \left\{\mu_{m}, \mu_{m, n}\right\}$. By continuity of $F_{m}$ and $F_{m, n}$, and using again the explicit expressions in Equations (36)-(37) (with general $m$ and $n$ inserted) as $k_{1}, k_{2} \rightarrow 1$, we have that

$$
\lim _{k_{1} \rightarrow \lambda^{+}} F_{m}\left(k_{1}, k_{2}\right)=F_{m}\left(\lambda, k_{2}\right) \leq \mu_{m}<\eta L_{1} \quad \text { and } \quad \lim _{k_{1} \rightarrow 1^{-}} F_{m}\left(k_{1}, k_{2}\right)=+\infty
$$

for every fixed $k_{2} \in[\lambda, 1]$, and

$$
\lim _{k_{2} \rightarrow \lambda^{+}} F_{m, n}\left(k_{1}, k_{2}\right)=F_{m, n}\left(k_{1}, \lambda\right) \leq \mu_{m, n}<\eta L_{2} \quad \text { and } \quad \lim _{k_{2} \rightarrow 1^{-}} F_{m, n}\left(k_{1}, k_{2}\right)=+\infty
$$

for every fixed $k_{1} \in[\lambda, 1]$. Then, Lemma 3 ensures that the level sets

$$
\left\{\left(k_{1}, k_{2}\right) \in Q: F_{m}\left(k_{1}, k_{2}\right)=\eta L_{1}\right\} \quad \text { and } \quad\left\{\left(k_{1}, k_{2}\right) \in Q: F_{m, n}\left(k_{1}, k_{2}\right)=\eta L_{2}\right\}
$$

respectively, are the graphs $k_{1}=f\left(k_{2}\right)$ and $k_{2}=g\left(k_{1}\right)$ of two continuous functions $f, g$ defined on $[\lambda, 1]$. The first graph joins a point on the segment $[\lambda, 1] \times\{1\}$ to a point on $[\lambda, 1] \times\{\lambda\}$, the latter one joins a point on $\{\lambda\} \times[\lambda, 1]$ to a point on $\{1\} \times[\lambda, 1]$, and therefore the two level sets must intersect in the interior of $Q$ at a point $\left(k_{1}, k_{2}\right)$, which thus solves the system in Equation (35). Then, Lines 4-7 of the system in Equation (33) fix the values of $a_{1}, a_{2}, a_{3}, a_{4}$, by taking $p$ as the unique integer such that the corresponding $a_{4}$ belongs to $\left(0, T_{2}\right]$. This completes the proof.

Remark 6. In the proof of Theorem 3, the sign of the function $F_{1}$ can be easily checked. Indeed, taking into account that $\theta_{k_{1}, k_{2}}^{+} \geq 1 / \sqrt{2}$, one has

$$
F_{1}\left(k_{1}, k_{2}\right)>2 \sqrt{2 k_{1}^{2}-1} \int_{\frac{\sqrt{2 k_{1}^{2}-1}}{k_{1} \sqrt{2}}}^{1} \frac{1}{\sqrt{1-t^{2}}}\left(\frac{1}{\sqrt{1-k_{1}^{2} t^{2}}}-\frac{1}{\sqrt{1-k_{1}^{2}\left(1-t^{2}\right)}}\right)>0 .
$$


On the contrary, the analysis of the sign of $\partial F_{1} / \partial k_{1}$ and $\partial F_{1,2} / \partial k_{2}$ over the set $A$ is rather involved and we could not perform it exactly. Therefore, we based our argument concerning the existence of the square $Q$ on the numerical evidence given by the plots of their graphs (see Figure 5), for which we used the software Wolfram MATHEMATICA $10 \cdot 4 \cdot 1$.
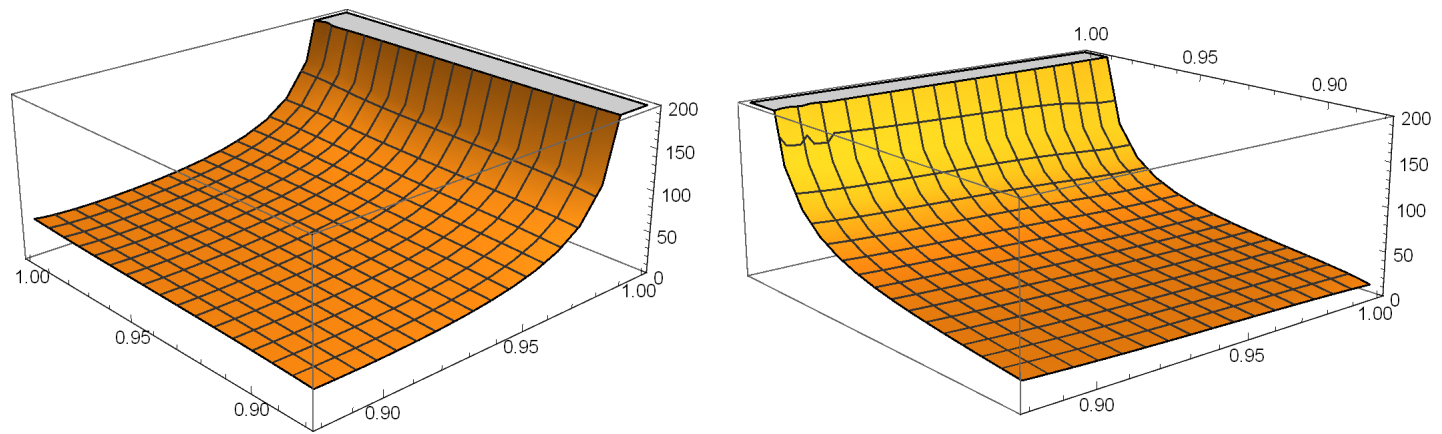

Figure 5. The functions $\partial F_{1} / \partial k_{1}$ and $\partial F_{1,2} / \partial k_{2}$ over the square $[\lambda, 1]^{2}$ with $\lambda=0.88$.

Author Contributions: All the authors contribute equally to this work

Funding: The research of D.N. and S.R. was funded in part by Departmental Project 2018-CONT-0127, University of Milano Bicocca.

Conflicts of Interest: The authors declare no conflict of interest. The funders had no role in the design of the study; in the collection, analyses, or interpretation of data and in the writing of the manuscript, or in the decision to publish the results.

\section{References}

1. Adami, R.; Serra, E.; Tilli, P. Nonlinear dynamics on branched structures and networks. Riv. Mat. Univ. Parma 2017, 8, 109-159.

2. Cacciapuoti, C.; Finco, D.; Noja, D. Ground state and orbital stability for the NLS equation on a general starlike graph with potentials. Nonlinearity 2017, 30, 3271-3303. [CrossRef]

3. Noja, D. Nonlinear Schrödinger equation on graphs: recent results and open problems. Philos. Trans. R. Soc. A 2014, 372, 20130002. [CrossRef] [PubMed]

4. Gnutzmann, S.; Waltner, D. Stationary waves on nonlinear quantum graphs. I. General framework and canonical perturbation theory, Phys. Rev. E 2016, 93, 032204. [PubMed]

5. Gnutzmann, S.; Waltner, D. Stationary waves on nonlinear quantum graphs. II. Application of canonical perturbation theory in basic graph structures. Phys. Rev. E 2016, 94, 062216. [CrossRef] [PubMed]

6. Marzuola, J.; Pelinovsky, D.E. Ground states on the dumbbell graph. Appl. Math. Res. Express 2016 1, $98-145$. [CrossRef]

7. Pelinovsky, D.E.; Schneider, G. Bifurcations of standing localized waves on periodic graphs. Ann. Henri Poincaré 2017, 18, 1185. [CrossRef]

8. Sobirov, Z.; Matrasulov, D.U.; Sabirov, K.K.; Sawada, S.; Nakamura, K. Integrable nonlinear Schrödinger equation on simple networks: Connection formula at vertices. Phys. Rev. E 2010, 81, 066602. [CrossRef] [PubMed]

9. Sabirov, K.K.; Sobirov, Z.A.; Babajanov, D.; Matrasulov, D.U. Stationary nonlinear Schrödinger equation on simplest graphs. Phys. Lett. A 2013, 377, 860-865. [CrossRef]

10. Adami, R.; Cacciapuoti, C.; Finco, D.; Noja, D. Stable standing waves for a NLS on star graphs as local minimizers of the constrained energy. J. Differ. Equ. 2016, 260, 7397-7415,

11. Adami, R.; Serra, E.; Tilli, P. NLS ground states on Graphs. Calc. Var. Partial Differ. Equ. 2015, 54, 743-761. [CrossRef]

12. Adami, R.; Serra, E.; Tilli, P. Threshold phenomena and existence results for NLS ground states on metric graphs. J. Funct. Anal. 2016, 271, 201-223. [CrossRef] 
13. Adami, R.; Serra, E.; Tilli, P. Negative Energy Ground States for the L2-Critical NLSE on Metric Graphs. Comm. Math. Phys. 2017, 352, 387-406. [CrossRef]

14. Berkolaiko, G.; Kuchment, P. Introduction to Quantum Graphs; Mathematical Surveys and Monographs 186; American Mathematical Society: Providence, RI, USA, 2013.

15. Noja, D.; Rolando, S.; Secchi, S. Standing waves for the NLS on the double-bridge graph and a rational-irrational dichotomy. J. Differ. Equ. 2019, 451, 147-178. [CrossRef]

16. Adami, R.; Serra, E.; Tilli, P. Multiple positive bound states for the subcritical NLS equation on metric graphs. Calc. Var. Partial Differ. Equ. 2019, 58. [CrossRef]

17. Cacciapuoti, C.; Finco, D.; Noja, D. Topology-induced bifurcations for the nonlinear Schrödinger equation on the tadpole graph. Phys. Rev. E 2015, 91, 013206. [CrossRef] [PubMed]

18. Noja, D.; Pelinovsky, D.; Shaikhova, G. Bifurcation and stability of standing waves in the nonlinear Schrödinger equation on the tadpole graph. Nonlinearity 2015, 28, 2343-2378. [CrossRef]

19. Lawden, D.F. Elliptic Functions and Applications; Springer: New York, NY, USA, 1989.

20. Olver, F.W.J.; Lozier, D.W.; Boisvert, R.F.; Clark, C.W. NIST Handbook of Mathematical Functions; Cambridge University Press: New York, NY, USA, 2010.

21. Niven, I. Diophantine Approximations; Interscience Tracts in Pure and Applied Mathematics No. 14; Interscience Publishers, Wiley \& Sons: New York, NY, USA, 1963.

(C) 2019 by the authors. Licensee MDPI, Basel, Switzerland. This article is an open access article distributed under the terms and conditions of the Creative Commons Attribution (CC BY) license (http://creativecommons.org/licenses/by/4.0/). 